TRANSACTIONS OF THE

AMERICAN MATHEMATICAL SOCIETY

Volume 360, Number 8, August 2008, Pages 4383-4408

S 0002-9947(08)04476-0

Article electronically published on March 20, 2008

\title{
CLASSES OF HARDY SPACES ASSOCIATED WITH OPERATORS, DUALITY THEOREM AND APPLICATIONS
}

\author{
LIXIN YAN
}

\begin{abstract}
Let $L$ be the infinitesimal generator of an analytic semigroup on $L^{2}\left(\mathbb{R}^{n}\right)$ with suitable upper bounds on its heat kernels. In Auscher, Duong, and McIntosh (2005) and Duong and Yan (2005), a Hardy space $H_{L}^{1}\left(\mathbb{R}^{n}\right)$ and a $\mathrm{BMO}_{L}\left(\mathbb{R}^{n}\right)$ space associated with the operator $L$ were introduced and studied. In this paper we define a class of $H_{L}^{p}\left(\mathbb{R}^{n}\right)$ spaces associated with the operator $L$ for a range of $p<1$ acting on certain spaces of Morrey-Campanato functions defined in New Morrey-Campanato spaces associated with operators and applications by Duong and Yan (2005), and they generalize the classical $H^{p}\left(\mathbb{R}^{n}\right)$ spaces. We then establish a duality theorem between the $H_{L}^{p}\left(\mathbb{R}^{n}\right)$ spaces and the Morrey-Campanato spaces in that same paper. As applications, we obtain the boundedness of fractional integrals on $H_{L}^{p}\left(\mathbb{R}^{n}\right)$ and give the inclusion between the classical $H^{p}\left(\mathbb{R}^{n}\right)$ spaces and the $H_{L}^{p}\left(\mathbb{R}^{n}\right)$ spaces associated with operators.
\end{abstract}

\section{INTRODUCTION}

This paper continues a line of study in [5], 17] and [18, where a class of the Hardy spaces $H_{L}^{1}\left(\mathbb{R}^{n}\right)$ and the $\mathrm{BMO}_{L}\left(\mathbb{R}^{n}\right)$ spaces associated with operators were introduced and developed, and they generalize the classical Hardy space $H^{1}\left(\mathbb{R}^{n}\right)$ and the BMO space. For the basic facts about the classical Hardy and BMO spaces on Euclidean spaces $\mathbb{R}^{n}$, see, for examples, [8], 21], 22], 30], [32] and 34].

Suppose that $L$ is a linear operator on $L^{2}\left(\mathbb{R}^{n}\right)$ which generates an analytic semigroup $e^{-t L}$ with a kernel $p_{t}(x, y)$ satisfying an upper bound, that is, there exist positive constants $m$ and $\epsilon$ such that for all $x, y \in \mathbb{R}^{n}$ and for all $t>0$,

$$
\left|p_{t}(x, y)\right| \leq \frac{c t^{\epsilon / m}}{\left(t^{1 / m}+|x-y|\right)^{n+\epsilon}} .
$$

In [5], Auscher, Duong and McIntosh defined a Hardy space $H_{L}^{1}\left(\mathbb{R}^{n}\right)$ associated with the operator $L$ as the class of all functions on $\mathbb{R}^{n}$ for which $S_{L}(f) \in L^{1}\left(\mathbb{R}^{n}\right)$ where

$$
S_{L}(f)(x)=\left(\int_{0}^{\infty} \int_{|y-x|<t}\left|Q_{t^{m}} f(y)\right|^{2} \frac{d y d t}{t^{n+1}}\right)^{1 / 2},
$$

and $Q_{t}=t L e^{-t L}$. They then obtained a molecular characterization for functions in $H_{L}^{1}\left(\mathbb{R}^{n}\right)$ by using the theory of tent spaces developed in [9] and [10].

Received by the editors July 15, 2005 and, in revised form, September 5, 2006.

2000 Mathematics Subject Classification. Primary 42B30, 42B35, 47B38.

The author was supported by NNSF of China (Grant No. 10571182/10771221) and by a grant from the Australia Research Council.

(C)2008 American Mathematical Society Reverts to public domain 28 years from publication 
A new function space $\mathrm{BMO}_{L}\left(\mathbb{R}^{n}\right)$ associated with the operator $L$ was introduced in [17. We say that a function $f$ (with suitable bounds on growth) is in $\mathrm{BMO}_{L}\left(\mathbb{R}^{n}\right.$ ) if

$$
\sup _{B} \frac{1}{|B|} \int_{B}\left|f(x)-e^{-t_{B} L} f(x)\right| d x<\infty,
$$

where $t_{B}=r_{B}^{m}$, and $r_{B}$ is the radius of the ball $B$. It was proved in [18] that if $L$ has a bounded holomorphic functional calculus on $L^{2}$ and the kernel $p_{t}(x, y)$ of the semigroup $e^{-t L}$ satisfies an upper bound (1.1), then the space $\operatorname{BMO}_{L^{*}}\left(\mathbb{R}^{n}\right)$ is the dual space of Hardy space $H_{L}^{1}\left(\mathbb{R}^{n}\right)$ in which $L^{*}$ denotes the adjoint operator of $L$. This gives a generalization of the duality of $H^{1}\left(\mathbb{R}^{n}\right)$ and $\operatorname{BMO}\left(\mathbb{R}^{n}\right)$ of Fefferman and Stein (21]). Indeed, a valid choice of $e^{-t L}$ in (1.2) and (1.3) is the Poisson semigroup $e^{-t \sqrt{\Delta}}$, which is defined by

$$
e^{-t \sqrt{\triangle}} f(x)=\int_{\mathbb{R}^{n}} p_{t}(x-y) f(y) d y, t>0, \text { where } p_{t}(x)=\frac{c_{n} t}{\left(t^{2}+|x|^{2}\right)^{(n+1) / 2}} .
$$

For this choice of $e^{-t \sqrt{\Delta}}$, the spaces $H_{\sqrt{\triangle}}^{1}\left(\mathbb{R}^{n}\right)$ and $\mathrm{BMO}_{\sqrt{\triangle}}\left(\mathbb{R}^{n}\right)$ coincide with the classical Hardy $H^{1}\left(\mathbb{R}^{n}\right)$ and BMO spaces, respectively ([5] and [17]).

For more properties of the space $H_{L}^{1}\left(\mathbb{R}^{n}\right)$ and the $\mathrm{BMO}_{L}\left(\mathbb{R}^{n}\right)$ space, we refer the reader to [5], 17, [18, [13] and [12].

Our concern in this paper is to introduce a class of Hardy spaces $H_{L}^{p}\left(\mathbb{R}^{n}\right)$ associated with $L$ for a range of $p<1$ and study their duals. The paper is organized as follows:

(i) To define the space $H_{L}^{p}\left(\mathbb{R}^{n}\right)$ for $p<1$, we use a space $\mathfrak{L}_{L}(\alpha, 2, s)$ of MorreyCampanato functions introduced in [19] that plays the role of the space $\mathcal{S}$ of test functions on $\mathbb{R}^{n}$ (Section 2). It turns out that given an operator $L$ with a bounded holomorphic functional calculus in $L^{2}\left(\mathbb{R}^{n}\right)$, which generates a semigroup with upper bounds (1.1) on its heat kernels, the Hardy space $H_{L}^{p}\left(\mathbb{R}^{n}\right)$ can be defined as the collection of all continuous linear functionals $f$ on $\mathfrak{L}_{L^{*}}\left(\frac{1}{p}-1,2,\left[\frac{n\left(\frac{1}{p}-1\right)}{m}\right]\right)$ satisfying $\mathcal{S}_{L}(f) \in L^{p}\left(\mathbb{R}^{n}\right)$. See Definition 3.1 below. Note that the spaces $H_{\sqrt{\Delta}}^{p}\left(\mathbb{R}^{n}\right)$ and $\mathfrak{L}_{\sqrt{\triangle}}(\alpha, 2, s)$ coincide with the classical $H^{p}\left(\mathbb{R}^{n}\right)$ and the Morrey-Campanato spaces $\mathrm{L}(\alpha, 2, s)\left(=\Lambda_{n \alpha}\left(\mathbb{R}^{n}\right)\right.$ of Lipschitz), respectively (Section 3 of [13]).

(ii) As in [5], we give a molecular decomposition for function $f$ in the $H_{L}^{p}\left(\mathbb{R}^{n}\right)$ spaces by using certain estimates on area integrals and tent spaces (see Proposition $3.3)$.

(iii) We establish a duality theorem, Theorem 4.1, which says that the dual space of $H_{L}^{p}\left(\mathbb{R}^{n}\right)$ is $\mathfrak{L}_{L^{*}}\left(\frac{1}{p}-1,2, s\right)$, by applying the results of previous sections, together with some estimates of the tent spaces and Carleson measures. With a choice of $e^{-t \sqrt{\triangle}}$, Theorem 4.1 gives the classical result of the duality of $H^{p}\left(\mathbb{R}^{n}\right)$ and $\mathrm{L}\left(\frac{1}{p}-1,2, s\right)$ for $p<1$ (see, for example, Theorem 2.7 of [34] and [16]).

(iv) In Sections 5 and 6 of this paper, we give applications, which include the boundedness of fractional integrals on the spaces $H_{L}^{p}\left(\mathbb{R}^{n}\right)$ and the inclusion between the classical spaces $H^{p}\left(\mathbb{R}^{n}\right)$ and the $H_{L}^{p}\left(\mathbb{R}^{n}\right)$ spaces associated with some differential operators.

We note that the idea of defining function spaces associated with certain operators is not new. In [3] and 2, the Hardy space associated with an elliptic second-order divergence operator $L$ was introduced by using the Poisson semigroup of $L$. In 20], Hardy spaces associated with Schrödinger operators were studied. In 
comparison with the classical $H^{p}\left(\mathbb{R}^{n}\right)$ spaces, an important feature of the $H_{L}^{p}\left(\mathbb{R}^{n}\right)$ spaces is that they tightly connect the operators considered, which may be an effective tool in the study of singular integral operators associated with the operator $L$; see [5, [12, 15, 17] and 18, for more details.

Throughout, the letter " $c$ " will denote (possibly different) constants that are independent of the essential variables.

\section{Preliminaries}

2.1. Holomorphic functional calculi of operators. We start with a review of some definitions of holomorphic functional calculi introduced by McIntosh [26]. Let $0 \leq \omega<\nu<\pi$. We define the closed sector in the complex plane $\mathbb{C}$

$$
S_{\omega}=\{z \in \mathbb{C}:|\arg z| \leq \omega\} \cup\{0\}
$$

and denote the interior of $S_{\omega}$ by $S_{\omega}^{0}$.

We employ the following subspaces of the space $H\left(S_{\nu}^{0}\right)$ of all holomorphic functions on $S_{\nu}^{0}$ :

$$
H_{\infty}\left(S_{\nu}^{0}\right)=\left\{b \in H\left(S_{\nu}^{0}\right):\|b\|_{\infty}<\infty\right\}
$$

where $\|b\|_{\infty}=\sup \left\{|b(z)|: z \in S_{\nu}^{0}\right\}$ and

$$
\Psi\left(S_{\nu}^{0}\right)=\left\{\psi \in H\left(S_{\nu}^{0}\right): \exists s>0,|\psi(z)| \leq c|z|^{s}\left(1+|z|^{2 s}\right)^{-1}\right\} .
$$

Given $0 \leq \omega<\pi$, a closed operator $L$ in $\mathrm{L}^{2}\left(\mathbb{R}^{n}\right)$ is said to be of type $\omega$ if $\sigma(L) \subset S_{\omega}$, and for each $\nu>\omega$, there exists a constant $c_{\nu}$ such that

$$
\left\|(L-\lambda I)^{-1}\right\|_{2,2} \leq c_{\nu}|\lambda|^{-1}, \quad \lambda \notin S_{\nu}
$$

If $L$ is of type $\omega$ and $\psi \in \Psi\left(S_{\nu}^{0}\right)$, we define $\psi(L) \in \mathcal{L}\left(\mathrm{L}^{2}, \mathrm{~L}^{2}\right)$ by

$$
\psi(L)=\frac{1}{2 \pi i} \int_{\Gamma}(L-\lambda I)^{-1} \psi(\lambda) d \lambda,
$$

where $\Gamma$ is the contour $\left\{\xi=r e^{ \pm i \theta}: r \geq 0\right\}$ parametrized clockwise around $S_{\omega}$, and $\omega<\theta<\nu$. Clearly, this integral is absolutely convergent in $\mathcal{L}\left(\mathrm{L}^{2}, \mathrm{~L}^{2}\right)$ (which is the class of all bounded linear operators on $\left.\mathrm{L}^{2}\left(\mathbb{R}^{n}\right)\right)$, and it is straightforward to show, using Cauchy's theorem, that the definition is independent of the choice of $\theta \in(\omega, \nu)$. If, in addition, $L$ is one-one and has dense range and if $b \in H_{\infty}\left(S_{\nu}^{0}\right)$, then $b(L)$ can be defined by

$$
b(L)=[\psi(L)]^{-1}(b \psi)(L) \quad \text { where } \quad \psi(z)=z(1+z)^{-2} .
$$

It can be shown that $b(L)$ is a well-defined linear operator in $\mathrm{L}^{2}\left(\mathbb{R}^{n}\right)$. We say that $L$ has a bounded $H_{\infty}$-calculus in $\mathrm{L}^{2}\left(\mathbb{R}^{n}\right)$ provided there exists $c_{\nu, 2}>0$ such that $b(L) \in \mathcal{L}\left(\mathrm{L}^{2}, \mathrm{~L}^{2}\right)$ and

$$
\|b(L)\|_{2,2} \leq c_{\nu, 2}\|b\|_{\infty}, \quad \forall b \in H_{\infty}\left(S_{\nu}^{0}\right) .
$$

An important feature of this functional calculus is the following convergence lemma.

Lemma 2.1 (Convergence lemma). Let $X$ be a complex Banach space. Given $0 \leq \omega<\nu \leq \pi$, let $L$ be an operator of type $\omega$ on $X$ which is one-to-one with dense domain and range. Suppose $\left\{f_{\alpha}\right\}$ is a uniformly bounded net in $H_{\infty}\left(S_{\nu}^{0}\right)$, which converges to $f \in H_{\infty}\left(S_{\nu}^{0}\right)$ uniformly on compact subsets of $S_{\nu}^{0}$, such that $\left\{f_{\alpha}(L)\right\}$ is a uniformly bounded net in the space $\mathcal{L}(X, X)$ of continuous linear operators on $X$. Then $f(L) \in \mathcal{L}(X, X), f_{\alpha}(L) u \rightarrow f(L) u$ for all $u \in X$ and $\|f(L)\| \leq \sup _{\alpha}\left\|f_{\alpha}(L)\right\|$. 
For the proof of Lemma 2.1, see [26] and [1].

2.2. Two more assumptions. Let $L$ be a linear operator of type $\omega$ on $L^{2}\left(\mathbb{R}^{n}\right)$ with $\omega<\pi / 2$, hence $L$ generates a holomorphic semigroup $e^{-z L}, 0 \leq|\operatorname{Arg}(z)|<\pi / 2-\omega$. Assume the following two conditions.

Assumption (a). Assume that for each $t>0$, the distribution kernel $p_{t}(x, y)$ of $e^{-t L}$ belongs to $L^{\infty}\left(\mathbb{R}^{n} \times \mathbb{R}^{n}\right)$ and satisfies the estimate

$$
\left|p_{t}(x, y)\right| \leq h_{t}(x, y)
$$

for $x, y \in \mathbb{R}^{n}$, where $h_{t}(x, y)$ is given by

$$
h_{t}(x, y)=t^{-n / m} g\left(\frac{|x-y|}{t^{1 / m}}\right),
$$

in which $m$ is a positive constant and $g$ is a positive, bounded, decreasing function satisfying

$$
\lim _{r \rightarrow \infty} r^{n+\epsilon} g(r)=0
$$

for some $\epsilon>0$.

Assumption (b). The operator $L$ is one-one and has dense range in $L^{2}\left(\mathbb{R}^{n}\right)$. Also, $L$ has a bounded $H_{\infty}$-calculus in $\mathrm{L}^{2}\left(\mathbb{R}^{n}\right)$.

Now, we give some consequences of the assumptions (a) and (b) which will be used later.

First, if $\left\{e^{-t L}\right\}_{t>0}$ is a bounded analytic semigroup on $L^{2}\left(\mathbb{R}^{n}\right)$ whose kernel $p_{t}(x, y)$ satisfies the estimates (2.1) and (2.2), then for any $k \in \mathbb{N}$, the time derivatives of $p_{t}$ satisfy

$$
\left|t^{k} \frac{\partial^{k} p_{t}(x, y)}{\partial t^{k}}\right| \leq \frac{c}{t^{n / m}} g\left(\frac{|x-y|}{t^{1 / m}}\right) \quad \text { for all } t>0 \text { and almost all } x, y \in \mathbb{R}^{n} .
$$

For each $k \in \mathbb{N}$, the function $g$ might depend on $k$ but it always satisfies (2.2). See Theorem 6.17 of [27] and 7].

Secondly, $L$ has a bounded $H_{\infty}$-calculus in $\mathrm{L}^{2}\left(\mathbb{R}^{n}\right)$ if and only if for any nonzero function $\psi \in \Psi\left(S_{\nu}^{0}\right), L$ satisfies the square function estimate and its reverse

$$
c_{1}\|f\|_{\mathrm{L}^{2}} \leq\left(\int_{0}^{\infty}\left\|\psi_{t}(L) f\right\|_{\mathrm{L}^{2}}^{2} \frac{d t}{t}\right)^{1 / 2} \leq c_{2}\|f\|_{\mathrm{L}^{2}}
$$

for some $0<c_{1} \leq c_{2}<\infty$, where $\psi_{t}(\xi)=\psi(t \xi)$. Note that different choices of $\nu>\omega$ and $\psi \in \Psi\left(S_{\nu}^{0}\right)$ lead to equivalent quadratic norms of $f$.

As noted in 26], positive self-adjoint operators satisfy the quadratic estimate (2.4). So do normal operators with spectra in a sector and maximal accretive operators. For definitions of these classes of operators, we refer the reader to [39].

2.3. Acting class of semigroup $\left\{e^{-t L}\right\}_{t>0}$. We now define the class of functions that the operators $e^{-t L}$ act upon. For any $\beta>0$, a function $f \in L_{\text {loc }}^{2}\left(\mathbb{R}^{n}\right)$ is said to be a function of $\beta$-type if $f$ satisfies

$$
\left(\int_{\mathbb{R}^{n}} \frac{|f(x)|^{2}}{1+|x|^{n+\beta}} d x\right)^{1 / 2} \leq c<\infty .
$$

We denote by $\mathcal{M}_{\beta}$ the collection of all functions of $\beta$-type. If $f \in \mathcal{M}_{\beta}$, the norm of $f$ in $\mathcal{M}_{\beta}$ is denoted by

$$
\|f\|_{\mathcal{M}_{\beta}}=\inf \{c \geq 0: \text { (2.5) holds }\} .
$$


It is easy to see that $\mathcal{M}_{\beta}$ is a Banach space under the norm $\|f\|_{\mathcal{M}_{\beta}}$. For any given operator $L$, we let

$$
\theta(L)=\sup \{\epsilon>0:(\underline{2.2}) \text { holds }\}
$$

and denote by

$$
\mathcal{M}= \begin{cases}\mathcal{M}_{\theta(L)}, & \text { if } \theta(L)<\infty ; \\ \bigcup_{\beta: 0<\beta<\infty} \mathcal{M}_{\beta}, & \text { if } \theta(L)=\infty .\end{cases}
$$

Note that if $L=\triangle$ is the Laplacian on $\mathbb{R}^{n}$, then $\theta(\triangle)=\infty$. When $L=\sqrt{\triangle}$, we have $\theta(\sqrt{\triangle})=1$.

Given an integer $s \in \mathbb{Z}^{+}$, for any $(x, t) \in \mathbb{R}_{+}^{n+1}$ and for $f \in \mathcal{M}$, we denote

(2.8) $P_{s, t} f(x)=f(x)-\left(I-e^{-t L}\right)^{s+1} f(x) \quad$ and $\quad Q_{s, t} f(x)=t^{s+1} L^{s+1} e^{-t L} f(x)$.

See [6] and [24]. In particular, if $s=0$, we denote by

$$
P_{t} f=P_{0, t} f=e^{-t L} f \quad \text { and } \quad Q_{t} f=Q_{0, t} f=t L e^{-t L} f .
$$

Since $f \in \mathcal{M}$, by the estimate (2.3) the operators $P_{s, t} f$ and $Q_{s, t} f$ are well defined. Moreover, the kernel $p_{s, t}(x, y)$ (resp. $\left.q_{s, t}(x, y)\right)$ of $P_{s, t}\left(\right.$ resp. $\left.Q_{s, t}\right)$ satisfies

$$
\left|p_{s, t^{m}}(x, y)\right| \leq c_{s} t^{-n} g\left(\frac{|x-y|}{t}\right) \quad\left(\text { resp. }\left|q_{s, t^{m}}(x, y)\right| \leq c_{s} t^{-n} g\left(\frac{|x-y|}{t}\right)\right),
$$

where the function $g$ satisfies the condition (2.2). This property is the same as the estimate (2.3).

2.4. The spaces $\mathfrak{L}_{L}(\alpha, q, s)$ associated with operators. The following definition was introduced in [19, which generalizes the classical Morrey-Campanato spaces $\mathrm{L}(\alpha, q, s)$. For the basic facts about the spaces $\mathrm{L}(\alpha, q, s)$, see [23] and [34].

Definition 2.2. Suppose $0 \leq \alpha<\theta(L) / n, 1 \leq q<\infty$ and $s \geq\left[\frac{n \alpha}{m}\right]$, the integral part of $\frac{n \alpha}{m}$. We say that $f \in \mathcal{M}$ is in $\mathfrak{L}_{L}(\alpha, q, s)$, the spaces of Morrey-Campanato type associated with $\left\{e^{-t L}\right\}_{t \geq 0}$, if there exists a positive constant $c$ such that for any ball $B$ of $\mathbb{R}^{n}$,

$$
\left[|B|^{-1} \int_{B}\left|f(x)-P_{s, t_{B}} f(x)\right|^{q} d x\right]^{1 / q} \leq c|B|^{\alpha},
$$

where $t_{B}=r_{B}^{m}$, and $r_{B}$ equals to the radius of the ball $B$.

The smallest bound $c$ satisfying condition (2.11) is then taken to be the norm of $f$ in this space and is denoted by $\|f\|_{\mathfrak{L}_{L}(\alpha, q, s)}$.

Note that $\left(\mathfrak{L}_{L}(\alpha, q, s),\|\cdot\|_{\mathfrak{L}(\alpha, q, s)}\right)$ is a seminormed vector space, with the seminorm vanishing on the space $\mathcal{K}_{(L, s)}$, defined by

$$
\mathcal{K}_{(L, s)}=\left\{f \in \mathcal{M}: P_{s, t} f(x)=f(x) \text { for almost all } x \in \mathbb{R}^{n} \text { for all } t>0\right\} .
$$

In this paper, the $\mathfrak{L}_{L}(\alpha, q, s)$ space is understood to be modulo $\mathcal{K}_{(L, s)}$. See Section 6 of [18] for a discussion of the dimensions of $\mathcal{K}_{(L, 0)}$ when $L$ is a second order elliptic operator of divergence form or a Schrödinger operator.

Now, we give some important properties of the spaces $\mathfrak{L}_{L}(\alpha, q, s)$ where $0<\alpha<$ $\theta(L) / n, 1 \leq q<\infty$ and $s \geq\left[\frac{n \alpha}{m}\right]$. 
First, for each $1 \leq q<\infty$, the space $\mathfrak{L}_{L}(0, q, 0)$ is a variant of the new $\mathrm{BMO}_{L}$ space introduced in [17, and it generalizes the classical BMO space. If $\theta(L)=\infty$, then the spaces $\mathfrak{L}_{L}(\alpha, q, s)$ are well defined for all $0 \leq \alpha<\infty, 1 \leq q<\infty$ and $s \geq\left[\frac{n \alpha}{m}\right]$. In particular, if $L$ is the Laplacian on $\mathbb{R}^{n}$, then the classical MorreyCampanato spaces $\mathrm{L}(\alpha, q, 2 s)$ coincide with our spaces $\mathfrak{L}_{\triangle}(\alpha, q, s)$. See [13].

Secondly, if $f \in \mathfrak{L}_{L}(\alpha, q, s)$ for $0<\alpha<\theta(L) / n, 1 \leq q<\infty$ and $s \geq\left[\frac{n \alpha}{m}\right]$, then

(a) for every $t>0$ and every $K>1$, there exists a constant $c>0$ such that for almost all $x \in \mathbb{R}^{n}$,

$$
\left|P_{s, t} f(x)-P_{s, K t} f(x)\right| \leq c(K t)^{n \alpha / m}\|f\|_{\mathfrak{L}_{L}(\alpha, q, s)} ;
$$

(b) For any $\delta>n \alpha$ and any $x \in \mathbb{R}^{n}$, there exists a constant $c_{\delta}$ which depends on $\delta$ such that

$$
\int_{\mathbb{R}^{n}} \frac{\left|f(y)-P_{s, t} f(y)\right|}{\left(t^{1 / m}+|x-y|\right)^{n+\delta}} d y \leq c_{\delta} t^{(n \alpha-\delta) / m}\|f\|_{\mathfrak{L}_{L}(\alpha, q, s)} .
$$

For the proofs of (a) and (b), see Propositions 2.5 and 2.7 of [19], respectively.

Proposition 2.3. Let $0<\alpha<\theta(L) / n, 1 \leq q<\infty$ and $s \geq\left[\frac{n \alpha}{m}\right]$.

(i) If $q_{t}(x, y)$ denotes the kernel of the operator $Q_{t}$, then for each $y \in \mathbb{R}^{n}$, $q_{t}(\cdot, y) \in \mathfrak{L}_{L}(\alpha, q, s)$. Similarly, for each $x \in \mathbb{R}^{n}, q_{t}(x, \cdot) \in \mathfrak{L}_{L^{*}}(\alpha, q, s)$.

(ii) If $f \in L^{2}\left(\mathbb{R}^{n}\right)$, then for each $t>0, Q_{t} f \in \mathfrak{L}_{L}(\alpha, q, s)$, and also, $Q_{t}^{*} f \in$ $\mathfrak{L}_{L^{*}}(\alpha, q, s)$.

Proof. In order to prove that for each $y \in \mathbb{R}^{n}, q_{t}(\cdot, y) \in \mathfrak{L}_{L}(\alpha, q, s)$, by Definition 2.2 it suffices to verify that for any ball $B$ of $\mathbb{R}^{n}$,

$$
\begin{aligned}
\int_{B}\left|q_{t}(x, y)-P_{s, t_{B}}\left(q_{t}(\cdot, y)\right)(x)\right|^{q} d x & =\int_{B}\left|\left(I-e^{-t_{B} L}\right)^{s+1}\left(q_{t}(\cdot, y)\right)(x)\right|^{q} d x \\
& \leq c|B|^{q \alpha+1},
\end{aligned}
$$

where $t_{B}=r_{B}^{m}$, and $r_{B}$ equals the radius of the ball $B$.

Let us prove (2.14). Noting that $I-e^{-t_{B} L}=-\int_{0}^{t_{B}} \frac{d}{d r} e^{-r L} d r=\int_{0}^{t_{B}} L e^{-r L} d r$, we have

$$
\begin{aligned}
\left(I-e^{-t_{B} L}\right)^{s+1}\left(t L e^{-t L}\right)= & \int_{0}^{t_{B}} \cdots \int_{0}^{t_{B}} \frac{t}{\left(t+r_{1}+\cdots+r_{s+1}\right)^{s+2}} \\
& \times Q_{s+1,\left(t+r_{1}+\cdots+r_{s+1}\right)} d r_{1} \cdots d r_{s+1} .
\end{aligned}
$$

From (2.10), the operator $\left(I-e^{-t_{B} L}\right)^{s+1}\left(t L e^{-t L}\right)$ has an associated kernel $K_{s, t}(x, y)$ which satisfies

$$
\begin{aligned}
\left|K_{s, t}(x, y)\right| & \\
\leq & c_{s+1} \int_{0}^{t_{B}} \cdots \int_{0}^{t_{B}} \frac{t}{\left(t+r_{1}+\cdots+r_{s+1}\right)^{s+2+\frac{n}{m}}} \\
& \times g\left(\frac{|x-y|}{\left(t+r_{1}+\cdots+r_{s+1}\right)^{1 / m}}\right) d r_{1} \cdots d r_{s+1} \\
\leq & c(s, t) \int_{0}^{t_{B}} \cdots \int_{0}^{t_{B}} \frac{t}{\left(t+r_{1}+\cdots+r_{s+1}\right)^{s+2}} d r_{1} \cdots d r_{s+1} \\
\leq & c^{\prime}(s, t) \int_{0}^{t_{B}} \frac{r^{s+1}}{(t+r)^{s+1}} \frac{d r}{r}
\end{aligned}
$$


for some constant $c(s, t)$ dependent on $s$ and $t$. We observe that $n \alpha \leq m(s+1)$, and then $\frac{r}{t+r} \leq c(t) r^{\frac{n \alpha}{m(s+1)}}$. Therefore,

$$
\left|K_{s, t}(x, y)\right| \leq c^{\prime}(s, t) c(t)^{s+1} \int_{0}^{t_{B}} r^{\frac{n \alpha}{m(s+1)}} \cdot(s+1) \frac{d r}{r} \leq c \int_{0}^{t_{B}} r^{\frac{n \alpha}{m}} \frac{d r}{r} \leq c t_{B}^{\frac{n \alpha}{m}} \leq c|B|^{\alpha},
$$

which gives the desired estimate (2.14), and then $q_{t}(\cdot, y) \in \mathfrak{L}_{L}(\alpha, q, s)$ for each $y \in \mathbb{R}^{n}$.

Similarly, for each $x \in \mathbb{R}^{n}, q_{t}(x, \cdot) \in \mathfrak{L}_{L^{*}}(\alpha, q, s)$. Also for $f \in L^{2}\left(\mathbb{R}^{n}\right)$, we have that $Q_{t} f \in \mathfrak{L}_{L}(\alpha, q, s)$ with $\left\|Q_{t} f\right\|_{\mathfrak{L}_{L}(\alpha, q, s)} \leq c\|f\|_{2}$ and $Q_{t}^{*} f \in \mathfrak{L}_{L^{*}}(\alpha, q, s)$ with $\left\|Q_{t}^{*} f\right\|_{\mathfrak{L}_{L^{*}}(\alpha, q, s)} \leq c\|f\|_{2}$.

We now introduce the dual space $\left(\mathfrak{L}_{L}(\alpha, q, s)\right)^{\prime}$ with $0<\alpha<\theta(L) / n, 1 \leq q<\infty$ and $s \geq\left[\frac{n \alpha}{m}\right]$ consisting of all linear functionals $\ell$ from $\mathfrak{L}_{L}(\alpha, q, s)$ to $\mathbb{C}$ with the property that there exists a finite constant $c$ such that for all $g \in \mathfrak{L}_{L}(\alpha, q, s)$,

$$
|\ell(g)| \leq c\|g\|_{\mathfrak{L}_{L}(\alpha, q, s)} .
$$

We denote by $\langle f, g\rangle$ the natural pairing of elements $f \in\left(\mathfrak{L}_{L}(\alpha, q, s)\right)^{\prime}$ and $g \in$ $\mathfrak{L}_{L}(\alpha, q, s)$. It follows from Proposition 2.3 that for all $f \in\left(\mathfrak{L}_{L}(\alpha, q, s)\right)^{\prime}$ with $0<\alpha<\theta(L) / n, 1 \leq q<\infty$ and $s \geq\left[\frac{n \alpha}{m}\right],\left\langle f, q_{t}(\cdot, y)\right\rangle$ is well defined. Similarly, for all $f \in\left(\mathfrak{L}_{L^{*}}(\alpha, q, s)\right)^{\prime}$ with $0<\alpha<\theta(L) / n, 1 \leq q<\infty$ and $s \geq\left[\frac{n \alpha}{m}\right],\left\langle f, q_{t}(x, \cdot)\right\rangle$ is well defined. In the following, we will denote $Q_{t} f(x)=\left\langle f, q_{t}(x, \cdot)\right\rangle$. Also, for any $f \in\left(\mathfrak{L}_{L^{*}}(\alpha, q, s)\right)^{\prime}$, we observe that for any $g \in L^{2}\left(\mathbb{R}^{n}\right)$,

$$
\left|\left\langle Q_{t} f, g\right\rangle\right|=\left|\left\langle f, Q_{t}^{*} g\right\rangle\right| \leq c\left\|Q_{t}^{*} g\right\|_{\mathfrak{L}_{L^{*}}(\alpha, q, s)} \leq c\|g\|_{2},
$$

and thus $Q_{t} f \in L^{2}\left(\mathbb{R}^{n}\right)$. These will often be used in the sequel.

\section{HaRdy Spaces $H_{L}^{p}\left(\mathbb{R}^{n}\right)$ ASSOciated With operators}

In what follows, $\mathbb{R}_{+}^{n+1}$ will denote the upper half-space in $\mathbb{R}^{n+1}$. The notation $\Gamma(x)=\left\{(y, t) \in \mathbb{R}_{+}^{n+1}:|x-y|<t\right\}$ denotes the standard cone (of aperture 1) with vertex $x \in \mathbb{R}^{n}$. For any closed subset $F \subset \mathbb{R}^{n}, \mathcal{R}(F)$ will be the union of all cones with vertices in $F$, i.e., $\mathcal{R}(F)=\bigcup_{x \in F} \Gamma(x)$. If $O$ is an open subset of $\mathbb{R}^{n}$, then the "tent" over $O$, denoted by $\widehat{O}$, is given as $\widehat{O}=\left[\mathcal{R}\left(O^{c}\right)\right]^{c}$.

3.1. Definition of Hardy spaces $H_{L}^{p}\left(\mathbb{R}^{n}\right)$. We continue with the assumption that the operator $L$ satisfies the assumptions (a) and (b) of Section 2.2. Given a function $f \in L^{1}\left(\mathbb{R}^{n}\right)$, the area integral function $\mathcal{S}_{L}(f)$ associated with an operator $L$ is defined by

$$
\mathcal{S}_{L}(f)(x)=\left(\int_{\Gamma(x)}\left|Q_{t^{m}} f(y)\right|^{2} \frac{d y d t}{t^{n+1}}\right)^{1 / 2} .
$$

It follows from the assumption (b) of $L$ that the area integral function $\mathcal{S}_{L}(f)$ is bounded on $L^{2}\left(\mathbb{R}^{n}\right)$. It was proved in Theorem 6 of $[5$ that there exist constants $c_{1}, c_{2}$ such that $0<c_{1} \leq c_{2}<\infty$ and

$$
c_{1}\|f\|_{p} \leq\left\|\mathcal{S}_{L}(f)\right\|_{p} \leq c_{2}\|f\|_{p}
$$

for all $f \in L^{p}\left(\mathbb{R}^{n}\right), 1<p<\infty$. See also [14] and 38.

By duality, the operator $S_{L^{*}}(f)$ also satisfies the estimate (3.2), where $L^{*}$ is the adjoint operator of $L$. 
3.1.1. The space $H_{L}^{1}\left(\mathbb{R}^{n}\right)$. The following definition was introduced in [5]. We say that $f \in L^{1}\left(\mathbb{R}^{n}\right)$ belongs to a Hardy space associated with an operator $L$ (abbreviated as $\left.H_{L}^{1}\right)$ if $S_{L}(f) \in L^{1}\left(\mathbb{R}^{n}\right)$, and define its norm by

$$
\|f\|_{H_{L}^{1}}=\left\|\mathcal{S}_{L}(f)\right\|_{L^{1}} .
$$

Note that if $L=\triangle$ is the Laplacian on $\mathbb{R}^{n}$, then the classical space $H^{1}\left(\mathbb{R}^{n}\right)$ coincides with the spaces $H_{\triangle}^{1}\left(\mathbb{R}^{n}\right)$ and $H_{\sqrt{\triangle}}^{1}\left(\mathbb{R}^{n}\right)$ and their norms are equivalent. See 21] and 30 .

3.1.2. The spaces $H_{L}^{p}\left(\mathbb{R}^{n}\right)$ with $p<1$. For a measurable function $g(y, t)$ defined on $\mathbb{R}_{+}^{n+1}$, we will denote

$$
\mathcal{A}(g)(x)=\left(\int_{\Gamma(x)}|g(y, t)|^{2} \frac{d y d t}{t^{n+1}}\right)^{1 / 2}
$$

and for $0<p \leq 1$,

$$
\mathcal{C}_{p}(g)(x)=\sup _{x \in B} \frac{1}{|B|^{\frac{1}{p}-\frac{1}{2}}}\left(\int_{\widehat{B}}|g(y, t)|^{2} \frac{d y d t}{t}\right)^{1 / 2} .
$$

Following [10, the "tent space" $T_{2}^{p}$ is defined as the space of functions $g$ such that $\mathcal{A}(g) \in L^{p}\left(\mathbb{R}^{n}\right)$, when $0<p<\infty$. The resulting equivalence classes are then equipped with the norm $\||g|\|_{T_{2}^{p}}=\|\mathcal{A}(g)\|_{p}$. When $p=\infty, T_{2}^{\infty}$ is the class of $g$ for which $\mathcal{C}_{1}(g) \in L^{\infty}\left(\mathbb{R}^{n}\right)$, and its norm is defined by $\left\|\mathcal{C}_{1}(g)\right\|_{\infty}$. For $0<p \leq 1$ we denote $T_{2}^{p, \infty}$ by

$$
T_{2}^{p, \infty}=\left\{g:\left\|\mathcal{C}_{p}(g)\right\|_{\infty}<\infty\right\} .
$$

Obviously, $T_{2}^{1, \infty}=T_{2}^{\infty}$.

We observe that $f \in H_{L}^{1}\left(\mathbb{R}^{n}\right)$ if and only if $Q_{t^{m}} f \in T_{2}^{1}$, i.e., $\mathcal{A}\left(Q_{t^{m}} f\right)=\mathcal{S}_{L}(f) \in$ $L^{1}$. From this point of view, we now introduce the Hardy spaces $H_{L}^{p}\left(\mathbb{R}^{n}\right)$ for $p<1$ associated with the semigroup $\left\{e^{-t L}\right\}_{t \geq 0}$.

Definition 3.1. Suppose $\frac{n}{n+\theta(L)}<p<1$ and $s_{0}=\left[\frac{n\left(\frac{1}{p}-1\right)}{m}\right]$. The generalized Hardy space $H_{L}^{p}\left(\mathbb{R}^{n}\right)$ associated with the semigroup $\left\{e^{-t L}\right\}_{t \geq 0}$ is the subspace of the dual space $\left(\mathfrak{L}_{L^{*}}\left(\frac{1}{p}-1,2, s_{0}\right)\right)^{\prime}$ of $\mathfrak{L}_{L^{*}}\left(\frac{1}{p}-1,2, s_{0}\right)$, defined as the completion of

$$
D_{p}:=\left\{f \in L^{2}\left(\mathbb{R}^{n}\right): \mathcal{A}\left(Q_{t^{m}} f\right) \in L^{p}\left(\mathbb{R}^{n}\right)\right\}, \quad \frac{n}{n+\theta(L)}<p<1,
$$

in the quasi-norm

$$
\|f\|_{H_{L}^{p}}=\left\|\mathcal{A}\left(Q_{t^{m}} f\right)\right\|_{L^{p}} .
$$

We will abuse language and say $\mathcal{A}\left(Q_{t^{m}} f\right)(x)$ is the area integral function associated with the semigroup $\left\{e^{-t L}\right\}_{t \geq 0}$, and still denoted by $\mathcal{S}_{L}(f)$.

Remarks. (i) Note first that smooth functions with compact support do not necessarily belong to $H_{L}^{p}\left(\mathbb{R}^{n}\right)$ in general. If $f \in H_{L}^{p}\left(\mathbb{R}^{n}\right)$, it follows from Theorem 4.1 below that $f$ satisfies the cancellation condition

$$
\int_{\mathbb{R}^{n}} f(x) g(x) d x=0
$$

for all $g \in \mathcal{K}_{\left(L^{*}, s_{0}\right)}$, where $\mathcal{K}_{\left(L^{*}, s_{0}\right)}$ is given by

$$
\mathcal{K}_{\left(L^{*}, s_{0}\right)}=\left\{g \in \mathcal{M}: P_{s_{0}, t}^{*} g(x)=g(x) \text { for almost all } x \in \mathbb{R}^{n} \text { for all } t>0\right\} .
$$


See Section 6 of [18 for a discussion of the dimensions of $\mathcal{K}_{\left(L^{*}, 0\right)}$ when $L$ is a second order elliptic operator of divergence form or a Schrödinger operator.

(ii) If $\theta(L)=\infty$, then the spaces $H_{L}^{p}\left(\mathbb{R}^{n}\right)$ are well defined for all $0<p \leq 1$. A typical example of $\theta(L)=\infty$ is when the kernel $p_{t}(x, y)$ of $e^{-t L}$ satisfies a Gaussian upper bound, that is,

$$
\left|p_{t}(x, y)\right| \leq \frac{C}{t^{n / 2}} e^{-c \frac{|x-y|^{2}}{t}}
$$

for $x, y \in \mathbb{R}^{n}$ and all $t>0$.

(iii) We now give a list of examples of $H_{L}^{p}\left(\mathbb{R}^{n}\right)$ in different settings.

$(\alpha)$ Let $p \leq 1$. The classical $H^{p}\left(\mathbb{R}^{n}\right)$ and the $H_{\triangle}^{p}\left(\mathbb{R}^{n}\right)$ spaces coincide, and their quasi-norms are equivalent. See 21] and 30.

( $\beta$ ) Let $A=\left(\left(a_{i j}(x)\right)_{1 \leq i, j \leq n}\right.$ be an $n \times n$ matrix with entries $a_{i j} \in L^{\infty}\left(\mathbb{R}^{n}, \mathbb{C}\right)$ satisfying $\operatorname{Re} \sum a_{i j}(x) \xi_{i} \xi_{j} \geq \lambda|\xi|^{2}$ for all $x \in \mathbb{R}^{n}, \xi=\left(\xi_{1}, \xi_{2}, \cdots, \xi_{n}\right) \in \mathbb{C}^{n}$ and some $\lambda>0$. We define a divergence form operator

$$
L f \equiv-\operatorname{div}(A \nabla f)
$$

which we interpret in the usual weak sense via a sesquilinear form.

Note that the Gaussian bound (3.5) on the heat kernel $e^{-t L}$ is true when $A$ has real entries, or when $n=1,2$ in the case of complex entries. See, for example, [4].

$(\gamma)$ Let $V \in L_{\text {loc }}^{1}\left(\mathbb{R}^{n}\right)$ be a nonnegative function on $\mathbb{R}^{n}$. The Schrödinger operator with potential $V$ is defined by

$$
L=-\Delta+V(x) \quad \text { on } \mathbb{R}^{n}, \quad n \geq 3 .
$$

From the Feynman-Kac formula, it is well known that the kernels $p_{t}(x, y)$ of the semigroup $e^{-t L}$ satisfy the estimate

$$
0 \leq p_{t}(x, y) \leq \frac{1}{(4 \pi t)^{n / 2}} e^{-\frac{|x-y|^{2}}{4 t}} .
$$

However, unless $V$ satisfies additional conditions, the heat kernel can be a discontinuous function of the space variables, and the Hölder continuous estimates may fail to hold. See, for example, [11] and 20].

3.2. The molecular structure for Hardy spaces $H_{L}^{p}\left(\mathbb{R}^{n}\right)$. Note that in [9, [10, the tent spaces give a natural and simple approach to the atomic decomposition of functions in the classical $H^{p}\left(\mathbb{R}^{n}\right)$ spaces by using the area integral functions and the connection with the theory of the Carleson measures. In this paper, we will adopt the same approach of tent spaces to obtain a molecular decomposition for Hardy spaces $H_{L}^{p}\left(\mathbb{R}^{n}\right)$. We now assume that

$$
\frac{n}{n+\theta(L)}<p<1, \quad s_{0}=\left[\frac{n\left(\frac{1}{p}-1\right)}{m}\right] \quad \text { and } \quad s \geq s_{0} .
$$

In the following, for any given $p$ as in (3.6), we let $\epsilon$ in (2.2) be a constant such that (i) $\epsilon>n\left(\frac{1}{p}-1\right)$ and thus $\frac{n}{n+\epsilon}<p$; (ii) $m\left(s_{0}+1\right)>\epsilon$ unless stated otherwise.

Following [10], a function $a(x, t)$ is called a $T_{2}^{p}$-atom, $0<p \leq 1$, if

(i) the function $a(t, x)$ is supported in $\widehat{B}$ (for some ball $B \subset \mathbb{R}^{n}$ ); and

(ii) $\int_{\widehat{B}}|a(t, x)|^{2} \frac{d x d t}{t} \leq|B|^{1-2 / p}$. 
Proposition 3.2. (a) Suppose $1<p<\infty$. The following inequality holds, whenever $f \in T_{2}^{p}$ and $g \in T_{2}^{p^{\prime}}$ with $\frac{1}{p}+\frac{1}{p^{\prime}}=1$,

$$
\int_{\mathbb{R}_{+}^{n+1}}|f(y, t) g(y, t)| \frac{d y d t}{t} \leq \int_{\mathbb{R}^{n}} \mathcal{A}(f)(x) \mathcal{A}(g)(x) d x .
$$

(b) Assume $0<p \leq 1$. Every element $f \in T_{2}^{p}$ can be written as $f=\sum \lambda_{j} a_{j}$, where $a_{j}$ are $T_{2}^{p}$ atoms, $\lambda_{j} \in \mathbb{C}$, and $\sum\left|\lambda_{j}\right|^{p} \leq c\|\| f \|_{T_{2}^{p}}^{p}$.

(c) If $0<p \leq 1$, then the dual space of $T_{2}^{p}$ is $T_{2}^{p, \infty}$. More precisely, the pairing $\langle f, g\rangle \rightarrow \int_{\mathbb{R}_{+}^{n+1}} f(x, t) g(x, t) \frac{d x d t}{t}$ realizes $T_{2}^{p, \infty}$ as equivalent to the dual of $T_{2}^{p}$.

Proof. For the proofs of (a) and (b), we refer to (5.1) and Proposition 5 of [10, respectively.

Let us show that $\ell \in\left(T_{2}^{p}\right)^{\prime}$ can be represented by a function $g \in T_{2}^{p, \infty}$. Following Theorem 1 of [10], we observe that if $K$ is a compact set of $\mathbb{R}_{+}^{n+1}$ and $f$ is supported in $K$ with $f \in L^{2}(K)$, then $f \in T_{2}^{p}$ with $\|\mid f\|_{T_{2}^{p}} \leq c_{K}\|f\|_{L^{2}(K)}$ for all $0<p \leq 1$. Thus $\ell$ induces a bounded linear function on $L^{2}(K)$ and is thus representable by a $g_{k} \in L^{2}(K)$. Taking an increasing family of such $K$ that exhausts $\mathbb{R}_{+}^{n+1}$ gives a function $g \in L_{\text {loc }}^{2}\left(\mathbb{R}_{+}^{n+1}\right)$ such that $\ell(f)=\int_{\mathbb{R}_{+}^{n+1}} f(x, t) g(x, t) \frac{d x d t}{t}$ whenever $f \in T_{2}^{p}$ and $f$ has compact support $K$. Testing $\ell$ against all possible atoms leads by the converse Schwartz's inequality to $|B|^{1-\frac{2}{p}} \int_{\widehat{B}}|g(x, t)|^{2} d x d t / t \leq\|\ell\|^{2}$ for all $B$, i.e. $\left\|\mathcal{C}_{p}(g)\right\|_{\infty} \leq\|\ell\|$ as desired. This representation of $\ell$ is then extendable to all of $T_{2}^{p}$, since the subspace of $f$ with compact support is dense in $T_{2}^{p}$. The proof of Proposition 3.2 is complete.

Let $m$ be the constant in (2.1). For any given $p<1$, we choose $s_{0}$ and $s$ the integers in (3.6). Let $c_{m, s}$ be a constant such that

$$
c_{m, s} \int_{0}^{\infty} t^{m(s+2)} e^{-2 t^{m}}\left(1-e^{-t^{m}}\right)^{s_{0}+1} d t / t=1 .
$$

We say that a function $\alpha(x)$ is a $(p, s)$-molecule if

$$
\alpha(x)=\pi_{L}(a)(x)=c_{m, s} \int_{0}^{\infty} Q_{s, t^{m}}\left(I-P_{s_{0}, t^{m}}\right)(a(t, \cdot))(x) \frac{d t}{t},
$$

where $a(t, x)$ is a $T_{2}^{p}$-atom supported in the tent $\widehat{B}$ of some ball $B \subset \mathbb{R}^{n}$, and $a(t, x)$ satisfies the condition $\int_{\widehat{B}}|a(t, x)|^{2} d x d t / t \leq|B|^{1-2 / p}$.

Proposition 3.3. Suppose $\frac{n}{n+\theta(L)}<p \leq 1$. For any $f \in H_{L}^{p}\left(\mathbb{R}^{n}\right) \cap L^{2}\left(\mathbb{R}^{n}\right)$, there exist $(p, s)$-molecules $\alpha_{k}(x)$ and numbers $\lambda_{k}(k=0,1,2, \cdots)$ such that

$$
f(x)=\sum_{k} \lambda_{k} \alpha_{k}(x) .
$$

The sequence $\lambda_{k}$ satisfies $\sum_{k}\left|\lambda_{k}\right|^{p} \leq c\|f\|_{H_{L}^{p}}^{p}$. Conversely, every sum (3.9) satisfies $\|f\|_{H_{L}^{p}}^{p} \leq c \sum_{k}\left|\lambda_{k}\right|^{p}$.

Proof. Let $c_{m, s}$ be a constant in (3.7). Consider the identity:

$$
1=c_{m, s} \int_{0}^{\infty}\left(t^{m(s+1)} z^{(s+1)} e^{-t^{m} z}\left(1-e^{-t^{m} z}\right)^{s_{0}+1}\right)\left(t^{m} z e^{-t^{m} z}\right) \frac{d t}{t},
$$


which is valid for all $z \neq 0$ in a sector $S_{\mu}^{0}$ with $\mu \in(\omega, \pi)$. As a consequence of $H_{\infty}$-functional calculus for $L$ and the convergence lemma 2.1, one has

$$
f(x)=c_{m, s} \int_{0}^{\infty} Q_{s, t^{m}}\left(I-P_{s_{0}, t^{m}}\right) Q_{t^{m}} f(x) \frac{d t}{t},
$$

where this integral converges strongly in $L^{2}\left(\mathbb{R}^{n}\right)$. See [1] and [26]. For any $f \in$ $H_{L}^{p}\left(\mathbb{R}^{n}\right) \cap L^{2}\left(\mathbb{R}^{n}\right)$, we let $F(x, t)=\left(Q_{t^{m}} f\right)(x)$. We then apply (b) of Proposition 3.2 to $Q_{t^{m}} f$ to obtain

$$
\begin{aligned}
f(x) & =c_{m, s} \int_{0}^{\infty} Q_{s, t^{m}}\left(I-P_{s_{0}, t^{m}}\right)\left(Q_{t^{m}} f\right)(x) \frac{d t}{t} \\
& =\sum_{k} \lambda_{k} c_{m, s} \int_{0}^{\infty} Q_{s, t^{m}}\left(I-P_{s_{0}, t^{m}}\right)\left(a_{k}(t, \cdot)\right)(x) \frac{d t}{t} \\
& =\sum_{k} \lambda_{k} \alpha_{k}(x),
\end{aligned}
$$

where the sequence $\lambda_{k}$ satisfies $\sum_{k}\left|\lambda_{k}\right|^{p} \leq c\|\| Q_{t^{m}}(f)\left\|\left.\right|_{T_{2}^{p}} ^{p} \leq c\right\| f \|_{H_{L}^{p}}^{p}$. This proved, when $f \in H_{L}^{p}\left(\mathbb{R}^{n}\right) \cap L^{2}\left(\mathbb{R}^{n}\right), f$ possesses a $(p, s)$-molecule decomposition.

Conversely, by the definition $H_{L}^{p}\left(\mathbb{R}^{n}\right)$, it suffices to verify that for any $(p, s)$ molecule $\alpha(x)$, we have

$$
\left\|\mathcal{S}_{L}(\alpha)\right\|_{p} \leq c
$$

where $c$ is a positive constant independent of $\alpha$.

Assume that $\alpha(x)=\pi_{L}(a)$ where $a=a(t, x)$ is a usual $T_{2}^{p}$-atom supported in $\widehat{B}$ (for some $B=B\left(z_{0}, r_{B}\right) \subset \mathbb{R}^{n}$ ). One writes

$$
\left\|\mathcal{S}_{L}(\alpha)\right\|_{L^{p}}^{p}=\int_{4 B}\left|\mathcal{S}_{L}(\alpha)(x)\right|^{p} d x+\int_{(4 B)^{c}}\left|\mathcal{S}_{L}(\alpha)(x)\right|^{p} d x=I+I I .
$$

Using Hölder's inequality and estimate (3.2), one obtains

$$
\begin{aligned}
\int_{4 B}\left|\mathcal{S}_{L}(\alpha)(x)\right|^{p} d x & \leq|4 B|^{1-\frac{p}{2}}\left\|\mathcal{S}_{L}\left(\pi_{L}(a)\right)\right\|_{L^{2}}^{p} \leq c|B|^{1-\frac{p}{2}}\left\|\pi_{L}(a)\right\|_{L^{2}}^{p} \\
& \leq c|B|^{1-\frac{p}{2}}\|\mid a\|_{T_{2}^{2}}^{p} \leq c .
\end{aligned}
$$

We now estimate the term $I I$. Firstly, we will show that there exists a constant $c>0$ such that for any $x \notin 4 B$,

$$
\mathcal{S}_{L}^{2}(\alpha)(x) \leq c r_{B}^{2 \epsilon}|B|^{2-\frac{2}{p}}\left|x-z_{0}\right|^{-2(n+\epsilon)} .
$$

Let us prove (3.12). For $k=0, \cdots, s_{0}+1$, we denote

$$
\Psi_{t, \nu}^{k}(L, s) f(y)=\left(t^{m}+(k+1) \nu^{m}\right)^{s+2}\left(\left.\frac{d^{s+2} P_{r}}{d r^{s+2}}\right|_{r=t^{m}+(k+1) \nu^{m}} f\right)(y) .
$$

By (2.3), the kernel $\psi_{t, \nu}^{k}(L, s)(x, y)$ of $\Psi_{t, \nu}^{k}(L, s)$ satisfies

$$
\left|\psi_{t, \nu}^{k}(L, s)(x, y)\right| \leq c_{k} \frac{(t+\nu)^{\epsilon}}{(t+\nu+|y-z|)^{n+\epsilon}} .
$$


Since $\left(I-P_{s_{0}, t^{m}}\right)=\sum_{k=0}^{s_{0}+1}(-1)^{k} C_{s_{0}+1}^{k} e^{-k t^{m} L}$, we obtain

$$
\begin{aligned}
\mathcal{S}_{L}^{2}(\alpha)(x)= & \left(\mathcal{S}_{L}\left(\int_{0}^{\infty} Q_{s, \nu^{m}}\left(I-P_{s_{0}, \nu^{m}}\right)(a(\nu, \cdot)) \frac{d \nu}{\nu}\right)\right)^{2}(x) \\
= & \int_{\Gamma(x)}\left[\int_{0}^{r_{B}} \sum_{k=0}^{s_{0}+1}(-1)^{k} C_{s_{0}+1}^{k} \frac{t^{m} \nu^{m(s+1)}}{\left(t^{m}+(k+1) \nu^{m}\right)^{s+2}}\right. \\
& \left.\times \Psi_{t, \nu}^{k}(L, s)(a(\nu, \cdot))(y) \frac{d \nu}{\nu}\right]^{2} \frac{d y d t}{t^{n+1}} \\
\leq & c \sum_{k=0}^{s_{0}+1} \int_{\Gamma(x)}\left[\int_{0}^{r_{B}} \int_{B} \frac{t^{m} \nu^{m(s+1)}}{\left(t^{m}+(k+1) \nu^{m}\right)^{s+2}}\left|\Psi_{t, \nu}^{k}(L, s)(y, z) a(\nu, z)\right| \frac{d z d \nu}{\nu}\right]^{2} \frac{d y d t}{t^{n+1}} \\
\leq & c \int_{0}^{r_{B}} \int_{|x-y|<t}\left[\int_{\widehat{B}} \frac{t^{m} \nu^{m(s+1)}}{(t+\nu)^{m(s+2)}} \frac{(t+\nu)^{\epsilon}|a(\nu, z)|}{(t+\nu+|y-z|)^{n+\epsilon}} \frac{d z d \nu}{\nu}\right]^{2} \frac{d y d t}{t^{n+1}} \\
& +c \int_{r_{B}}^{\infty} \int_{|x-y|<t}\left[\int_{\widehat{B}} \frac{t^{m} \nu^{m(s+1)}}{(t+\nu)^{m(s+2)}} \frac{(t+\nu)^{\epsilon}|a(\nu, z)|}{(t+\nu+|y-z|)^{n+\epsilon}} \frac{d z d \nu}{\nu}\right]^{2} \frac{d y d t}{t^{n+1}} \\
= & I I_{1}+I I_{2}, \quad \text { respectively. }
\end{aligned}
$$

We only consider term $I I_{2}$ since the estimate of term $I I_{1}$ is much simpler. For $x \notin 4 B$ and $t \geq r_{B}$, we set $B=B_{1} \cup B_{2}$, where $B_{1}=B \cap\left\{z:|y-z| \leq \frac{\left|x-z_{0}\right|}{2}\right\}$. For any $z \in B_{1}$ and $|y-x|<t$, we have

$$
\left|x-z_{0}\right| \leq|y-x|+|y-z|+\left|z-z_{0}\right| \leq t+\frac{\left|x-z_{0}\right|}{2}+r_{B} \leq 2 t+\frac{\left|x-z_{0}\right|}{2}
$$

which implies $t \geq\left|x-z_{0}\right| / 4$, and then $(t+s+|y-z|) \geq\left|x-z_{0}\right| / 4$. Obviously, for any $z \in B_{2}$ and $|y-x|<t$, we also have $(t+s+|y-z|) \geq\left|x-z_{0}\right| / 2$. Those, together with

$$
(t+\nu)^{\epsilon} \cdot \frac{t^{m} \nu^{m(s+1)}}{(t+\nu)^{m(s+2)}} \leq t^{\epsilon}\left(\frac{\nu}{t}\right)^{m(s+1)}
$$

and $m(s+1)>\epsilon$, give

$$
\begin{aligned}
I I_{2} & \leq c \int_{r_{B}}^{\infty} \int_{|x-y|<t}\left[\int_{\widehat{B}} \nu^{m(s+1)} t^{\epsilon-m(s+1)}|a(\nu, z)| \frac{d z d \nu}{\nu}\right]^{2} \frac{d y d t}{t^{n+1}}\left|x-z_{0}\right|^{-2(n+\epsilon)} \\
& \leq c|B| \int_{r_{B}}^{\infty} \int_{0}^{r_{B}} \nu^{2 m(s+1)} t^{2 \epsilon-2 m(s+1)} \frac{d \nu d t}{\nu t} \int_{\widehat{B}}|a(\nu, z)|^{2} \frac{d z d \nu}{\nu}\left|x-z_{0}\right|^{-2(n+\epsilon)} \\
& \leq c|B|^{2-\frac{2}{p}} \int_{r_{B}}^{\infty} \int_{0}^{r_{B}} \nu^{2 m(s+1)} t^{2 \epsilon-2 m(s+1)} \frac{d \nu d t}{\nu t}\left|x-z_{0}\right|^{-2(n+\epsilon)} \\
& \leq c r_{B}^{2 \epsilon+2 n-\frac{2 n}{p}}\left|x-z_{0}\right|^{-2(n+\epsilon)} .
\end{aligned}
$$

Estimate (3.12) then follows readily. Since $p(n+\epsilon)>n$, we obtain

$$
\int_{(4 B)^{c}}\left|\mathcal{S}_{L}(\alpha)(x)\right|^{p} d x \leq c r_{B}^{p \epsilon+n p-n} \int_{(4 B)^{c}}\left|x-z_{0}\right|^{-(n+\epsilon) p} d x \leq c .
$$

Combining estimates of $I$ and $I I$, we obtain (3.11), and then the proof of Proposition 3.3 is complete. 
Now, let $T_{2, c}^{p}$ be the set of all $f \in T_{2}^{p}$ with compact support in $\mathbb{R}_{+}^{n+1}$. Consider the operator $\pi_{L}$ initially defined on $T_{2, c}^{p}$ by

$$
\pi_{L}(f)(x)=c_{m, s} \int_{0}^{\infty} Q_{s, t^{m}}\left(I-P_{s_{0}, t^{m}}\right)(f(\cdot, t))(x) \frac{d t}{t},
$$

where $c_{m, s}$ is a constant in (3.7). Note that for any compact set $K$ in $\mathbb{R}_{+}^{n+1}$,

$$
\int_{K}|f(x, t)|^{2} d x d t \leq c(K, p)\|\mathcal{A}(f)\|_{p}^{2}
$$

for all $p>0$. See page 306 of [10. This and the estimate (2.4) imply that the integral (3.14) is well defined, and $\pi_{L}(f) \in L^{2}\left(\mathbb{R}^{n}\right)$ for all $f \in T_{2, c}^{p}$.

Lemma 3.4. The operator $\pi_{L}$, initially defined on $T_{2, c}^{p}$, extends to a bounded linear operator from

(a) $T_{2}^{p}$ to $L^{p}$ if $1<p<\infty$;

(b) $T_{2}^{p}$ to $H_{L}^{p}$ if $\frac{n}{n+\theta(L)}<p \leq 1$.

Proof. The property (b) is contained in the second part of Proposition 3.3. We now verify the property (a). From Proposition 3.2 and estimate (3.2), we have

$$
\begin{aligned}
\left|\int_{\mathbb{R}^{n}} \pi_{L}(f)(x) g(x) d x\right| & \leq c\left|\int_{\mathbb{R}_{+}^{n+1}} f(x, t)\left(Q_{s, t^{m}}\left(I-P_{s_{0}, t^{m}}\right)\right)^{*} g(x) \frac{d x d t}{t}\right| \\
& \leq c\left|\int_{\mathbb{R}^{n}} \mathcal{A}(f)(x) \mathcal{A}\left(\left(Q_{s, t^{m}}\left(I-P_{s_{0}, t^{m}}\right)\right)^{*} g\right)(x) d x\right| \\
& \leq c\|\mathcal{A}(f)\|_{p}\left\|\mathcal{A}\left(\left(Q_{s, t^{m}}\left(I-P_{s_{0}, t^{m}}\right)\right)^{*} g\right)\right\|_{p^{\prime}} \\
& \leq c\|f \mid\|\left\|_{T_{2}^{p}}\right\| g \|_{p^{\prime}}
\end{aligned}
$$

for any $g \in L^{p^{\prime}}, \frac{1}{p}+\frac{1}{p^{\prime}}=1$. Hence, we obtain $\left\|\pi_{L}(f)\right\|_{p} \leq c\left|\|f \mid\|_{T_{2}^{p}}\right.$.

We next state the following $H_{L}^{p}$-estimate for functions in the space $H_{L}^{p}\left(\mathbb{R}^{n}\right)$. For its proof, it is similar to that of the second part of Proposition 3.3. We omit details here.

Proposition 3.5. Suppose $\frac{n}{n+\theta(L)}<p \leq 1$ and $s_{0}=\left[\frac{n\left(\frac{1}{p}-1\right)}{m}\right]$. For any $L^{2}$-function $f$ supported on $B$, the function $\left(I-P_{s_{0}, r_{B}^{m}}\right) f$ belongs to $H_{L}^{p}\left(\mathbb{R}^{n}\right)$, and there exists a positive constant $c$ such that

$$
\left\|\left(I-P_{s_{0}, r_{B}^{m}}\right) f\right\|_{H_{L}^{p}} \leq c|B|^{\frac{1}{p}-\frac{1}{2}}\|f\|_{L^{2}},
$$

where $r_{B}$ is the radius of the ball $B$.

\section{Duality of $H_{L}^{p}$ And $\mathfrak{L}_{L}(\alpha, 2, s)$ Spaces}

In this section, we assume that the operator $L$ satisfies the assumptions (a) and (b) of Section 2. It was proved in Theorem 3.1 of [18] that the dual space of $H_{L}^{1}\left(\mathbb{R}^{n}\right)$ is the space $\mathrm{BMO}_{L^{*}}\left(\mathbb{R}^{n}\right)$ in which $L^{*}$ is the adjoint operator of $L$. The aim of this section is to prove the following theorem.

Theorem 4.1. Suppose $\frac{n}{n+\theta(L)}<p<1$ and $s_{0}=\left[\frac{n\left(\frac{1}{p}-1\right)}{m}\right]$. Then, the dual space of the $H_{L}^{p}\left(\mathbb{R}^{n}\right)$ space is the $\mathfrak{L}_{L^{*}}\left(\frac{1}{p}-1,2, s_{0}\right)$ space, in the following sense. 
(i) Suppose $f \in \mathfrak{L}_{L^{*}}\left(\frac{1}{p}-1,2, s_{0}\right)$. Then the linear functional $\ell$ given by

$$
\ell(g)=\int_{\mathbb{R}^{n}} f(x) g(x) d x
$$

initially defined on the dense subspace $H_{L}^{p}\left(\mathbb{R}^{n}\right) \cap L^{2}\left(\mathbb{R}^{n}\right)$, has a unique extension to $H_{L}^{p}\left(\mathbb{R}^{n}\right)$.

(ii) Conversely, every continuous linear functional $\ell$ on the $H_{L}^{p}\left(\mathbb{R}^{n}\right)$ space can be realized as above, i.e., there exists $f \in \mathfrak{L}_{L^{*}}\left(\frac{1}{p}-1,2, s_{0}\right)$ such that (4.1) holds and $\|f\|_{\mathfrak{L}_{L^{*}\left(\frac{1}{p}-1,2, s_{0}\right)}} \leq c\|\ell\|$.

Recall that a measure $\mu$ in $\mathbb{R}_{+}^{n+1}$ is a Carleson measure $V^{\beta}$ of order of $\beta \geq 1$ if there is a positive constant $c$ such that for each ball $B$ on $\mathbb{R}^{n}$,

$$
\mu(\widehat{B}) \leq c|B|^{\beta},
$$

where $\widehat{B}$ is the tent over $B$. The smallest bound $c$ in (4.2) is defined to be the norm of $\mu$, and denoted by $\|\mid \mu\|_{V^{\beta}}$. See, for example, page 338, Chapter XV, of [33].

Proposition 4.2. Suppose $0<\alpha<\theta(L) / n$ and $s \geq s_{0}=\left[\frac{n \alpha}{m}\right]$. If $f \in \mathfrak{L}_{L}\left(\alpha, 2, s_{0}\right)$, then the measure

$$
\mu_{f}(x, t)=\left|Q_{s, t^{m}}\left(\mathcal{I}-P_{s_{0}, t^{m}}\right) f(x)\right|^{2} \frac{d x d t}{t}
$$

is a Carleson measure $V^{2 \alpha+1}$ with $\left\|\mid \mu_{f}\right\|\left\|_{V^{(2 \alpha+1)}} \leq c\right\| f \|_{\mathfrak{L}_{L}\left(\alpha, 2, s_{0}\right)}^{2}$.

Proof. Given $0<\alpha<\theta(L) / n$, we let $\epsilon$ in (2.2) be the constant such that $n \alpha<\epsilon<$ $\theta(L)$ and $m(s+1)>\epsilon$. In order to prove Proposition 4.2, it suffices to prove that there exists a positive constant $c>0$ such that for any ball $B=B\left(x_{B}, r_{B}\right)$ on $\mathbb{R}^{n}$,

$$
\iint_{\widehat{B}}\left|Q_{s, t^{m}}\left(I-P_{s_{0}, t^{m}}\right) f(x)\right|^{2} \frac{d x d t}{t} \leq c|B|^{2 \alpha+1}\|f\|_{\mathfrak{L}_{L}\left(\alpha, 2, s_{0}\right)}^{2}
$$

Note that $\left(I-P_{s_{0}, t^{m}}\right)=\left(I-P_{s_{0}, t^{m}}\right)\left(I-P_{s_{0}, r_{B}^{m}}\right)+P_{s_{0}, r_{B}^{m}}\left(I-P_{s_{0}, t^{m}}\right)$. Hence, estimate (4.3) will follow from the following estimates (4.4) and (4.5):

$$
\iint_{\widehat{B}}\left|Q_{s, t^{m}}\left(I-P_{s_{0}, t^{m}}\right)\left(I-P_{s_{0}, r_{B}^{m}}\right) f(x)\right|^{2} \frac{d x d t}{t} \leq c|B|^{2 \alpha+1}\|f\|_{\mathfrak{L}_{L}\left(\alpha, 2, s_{0}\right)}^{2}
$$

and

$$
\iint_{\widehat{B}}\left|Q_{s, t^{m}} P_{s_{0}, r_{B}^{m}}\left(I-P_{s_{0}, t^{m}}\right) f(x)\right|^{2} \frac{d x d t}{t} \leq c|B|^{2 \alpha+1}\|f\|_{\mathfrak{L}_{L}\left(\alpha, 2, s_{0}\right)}^{2} .
$$

To prove (4.4), let us introduce the square function $\mathcal{G} f$, given by

$$
\mathcal{G}(f)(x)=\left(\int_{0}^{\infty}\left|Q_{s, t^{m}}\left(I-P_{s_{0}, t^{m}}\right) f(x)\right|^{2} \frac{d t}{t}\right)^{1 / 2} .
$$


From (2.4), the function $\mathcal{G}(f)$ is bounded on $L^{2}\left(\mathbb{R}^{n}\right)$. Let $b_{1}=\left(I-P_{s_{0}, r_{B}^{m}}\right) f \chi_{2 B}$, and $b_{2}=\left(I-P_{s_{0}, r_{B}^{m}}\right) f \chi_{(2 B)^{c}}$. Using property $(2.12)$ of $\mathfrak{L}_{L}\left(\alpha, 2, s_{0}\right)$, we obtain

$$
\begin{aligned}
\iint_{\widehat{B}}\left|Q_{s, t^{m}}\left(I-P_{s_{0}, t^{m}}\right) b_{1}(x)\right|^{2} \frac{d x d t}{t} \\
\leq\left\|\mathcal{G}\left(b_{1}\right)\right\|_{2}^{2} \\
\leq c\left\|b_{1}\right\|_{L^{2}\left(\mathbb{R}^{n}\right)}^{2} \\
\quad=c \int_{2 B}\left|\left(I-P_{s_{0}, r_{B}^{m}}\right) f(x)\right|^{2} d x \\
\quad \leq c\left(\int_{2 B}\left|\left(I-P_{s_{0}, r_{2 B}^{m}}^{m}\right) f(x)\right|^{2} d x+|B| \cdot \sup _{x \in 2 B}\left|P_{s_{0}, r_{B}^{m}} f(x)-P_{s_{0}, r_{2 B}^{m}} f(x)\right|^{2}\right) \\
(4.6) \quad \leq c|B|^{2 \alpha+1}\|f\|_{\mathfrak{L}_{L}\left(\alpha, 2, s_{0}\right)}^{2} .
\end{aligned}
$$

On the other hand, for any $x \in B$ and $y \in(2 B)^{c}$, one has $|x-y| \geq r_{B}$. And then by estimate (2.10) and property (iv) of $\mathfrak{L}_{L}\left(\alpha, 2, s_{0}\right)$,

$$
\begin{aligned}
\left|Q_{s, t^{m}}\left(I-P_{s_{0}, t^{m}}\right) b_{2}(x)\right| & \leq c \int_{\mathbb{R}^{n} \backslash 2 B} \frac{t^{\epsilon}}{(t+|x-y|)^{n+\epsilon}}\left|\left(\mathcal{I}-P_{s_{0}, r_{B}^{m}}\right) f(y)\right| d y \\
& \leq c\left(\frac{t}{r_{B}}\right)^{\epsilon} \int_{\mathbb{R}^{n}} \frac{r_{B}^{\epsilon}}{\left(r_{B}+|x-y|\right)^{n+\epsilon}}\left|\left(I-P_{s_{0}, r_{B}^{m}}\right) f(y)\right| d y \\
& \leq c|B|^{2 \alpha}\left(\frac{t}{r_{B}}\right)^{\epsilon}\|f\|_{\mathfrak{L}_{L}\left(\alpha, 2, s_{0}\right)}
\end{aligned}
$$

since $n \alpha<\epsilon$. Therefore,

$$
\begin{aligned}
\iint_{\widehat{B}}\left|Q_{s_{0}, t^{m}}\left(I-P_{s_{0}, t^{m}}\right) b_{2}(x)\right|^{2} \frac{d x d t}{t} & \leq c|B|^{2 \alpha} \frac{1}{r_{B}^{2 \epsilon}} \iint_{\hat{B}} t^{2 \epsilon} \frac{d x d t}{t}\|f\|_{\mathfrak{L}_{L}\left(\alpha, 2, s_{0}\right)}^{2} \\
& \leq c|B|^{2 \alpha+1}\|f\|_{\mathfrak{L}_{L}\left(\alpha, 2, s_{0}\right)}^{2} .
\end{aligned}
$$

This, together with the estimate (4.6), gives the estimate (4.4).

Let us prove (4.5). For $k=1,2, \cdots, s_{0}+1$, we denote by

$$
\Psi_{t, r_{B}}^{k} f(x)=\left(k r_{B}^{m}+t^{m}\right)^{s+1}\left(\left.\frac{d^{s+1} P_{\nu}}{d \nu^{s+1}}\right|_{\nu=k r_{B}^{m}+t^{m}} f\right)(x) .
$$

From (2.10), the kernel $\Psi_{t, r_{B}}^{k}(x, y)$ of $\Psi_{t, r_{B}}^{k}$ satisfies

$$
\left|\Psi_{t, r_{B}}^{k}(x, y)\right| \leq c_{k} \frac{r_{B}^{\epsilon}}{\left(r_{B}+t+|x-y|\right)^{n+\epsilon}} .
$$

Since $P_{s_{0}, r_{B}^{m}} f(x)=\sum_{k=1}^{s_{0}+1}(-1)^{k+1} C_{s_{0}+1}^{k} e^{-k r_{B}^{m} L} f(x)$, from property (2.13) of $\mathfrak{L}_{L}\left(\alpha, 2, s_{0}\right)$ together with $n \alpha<\epsilon$, we have

$$
\begin{aligned}
\mid Q_{s, t^{m}} P_{s_{0}, r_{B}^{m}} & \left(I-P_{s_{0}, t^{m}}\right) f(x) \mid \\
& =\left|\sum_{k=1}^{s_{0}+1}(-1)^{k+1} C_{s_{0}+1}^{k} \frac{t^{m(s+1)}}{\left(k r_{B}^{m}+t^{m}\right)^{s+1}} \Psi_{t, r_{B}}^{k}\left(I-P_{s_{0}, t^{m}}\right) f(x)\right| \\
& \leq c\left(\frac{t}{r_{B}}\right)^{m(s+1)-\epsilon} \int_{\mathbb{R}^{n}} \frac{t^{\epsilon}}{(t+|x-y|)^{n+\epsilon}}\left|\left(I-P_{s_{0}, t^{m}}\right) f(y)\right| d y \\
& \leq c t^{n \alpha / m}\left(\frac{t}{r_{B}}\right)^{m(s+1)-\epsilon}\|f\|_{\mathfrak{L}_{L}\left(\alpha, 2, s_{0}\right)}, \quad \forall 0<t \leq t_{B} .
\end{aligned}
$$


Therefore, by using the condition $m(s+1)>\epsilon$,

$$
\begin{aligned}
\iint_{\widehat{B}} & \left.\mid Q_{s, t^{m}}\left(I-P_{s_{0}, t^{m}}\right) P_{s_{0}, r_{B}^{m}}\right)\left.f(x)\right|^{2} \frac{d x d t}{t} \\
& \leq c \iint_{\hat{B}} t^{2 n \alpha / m-1}\left(\frac{t}{r_{B}}\right)^{m(s+1)-\epsilon} d x d t\|f\|_{\mathfrak{L}_{L}\left(\alpha, 2, s_{0}\right)}^{2} \\
& \leq c|B|^{2 \alpha+1}\|f\|_{\mathfrak{L}_{L}\left(\alpha, 2, s_{0}\right)}^{2},
\end{aligned}
$$

which gives the proof of (4.3), and therefore the proof of Proposition 4.2.

Proof of Theorem 4.1. Suppose $f \in \mathfrak{L}_{L^{*}}\left(\frac{1}{p}-1,2, s_{0}\right)$ and $b$ is a $(p, s)$-molecule of $H_{L}^{p}\left(\mathbb{R}^{n}\right)$. Without loss of generality, we assume that

$$
b(x)=c_{m, s} \int_{0}^{\infty} Q_{s, t^{m}}\left(I-P_{s_{0}, t^{m}}\right)(a(t, \cdot))(x) \frac{d t}{t},
$$

where $a(t, x)$ is a usual $T_{2}^{p}$-atom supported in $\widehat{B}$ (for some $B=B\left(z_{0}, r_{B}\right) \subset \mathbb{R}^{n}$ ), and $c_{m, s}$ is the constant in (3.7). We can apply the same argument as in Theorem 5.1 of [18 to obtain the following identity:

$$
\int_{\mathbb{R}^{n}} b(x) f(x) d x=c_{m, s} \int_{\mathbb{R}_{+}^{n+1}} a(t, x) Q_{s, t^{m}}^{*}\left(I-P_{s_{0}, t^{m}}^{*}\right) f(x) \frac{d x d t}{t} .
$$

The details are omitted here. This, together with Proposition 4.2, shows that

$$
\begin{aligned}
\left|\int_{\mathbb{R}^{n}} b(x) f(x) d x\right| & \leq c\left\||a \||_{T_{2}^{2}}\left(\int_{\widehat{B}}\left|Q_{s, t^{m}}^{*}\left(I-P_{s_{0}, t^{m}}^{*}\right) f(x)\right|^{2} \frac{d x d t}{t}\right)^{1 / 2}\right. \\
& \leq c|B|^{\frac{1}{2}-\frac{1}{p}}|B|^{\frac{1}{p}-1+\frac{1}{2}} \\
& \leq c .
\end{aligned}
$$

For any $g \in H_{L}^{p}\left(\mathbb{R}^{n}\right) \cap L^{2}\left(\mathbb{R}^{n}\right)$, it follows from Proposition 3.3 that there exist $(p, s)$ molecules $\alpha_{k}(x)$ and numbers $\lambda_{k}(k=0,1,2, \cdots)$ such that $g(x)=\sum_{k} \lambda_{k} \alpha_{k}(x)$. The sequence $\lambda_{k}$ satisfies $\sum_{k}\left|\lambda_{k}\right|^{p} \leq c\|g\|_{H_{L}^{p}}^{p}$. Hence by (4.7) we obtain

$$
\begin{aligned}
\left|\int_{\mathbb{R}^{n}} f(x) g(x) d x\right| & \leq \sum_{k}\left|\lambda_{k}\right|\left|\int_{\mathbb{R}^{n}} \alpha_{k}(x) f(x) d x\right| \leq c \sum_{k}\left|\lambda_{k}\right| \\
& \leq c\left(\sum_{k}\left|\lambda_{k}\right|^{p}\right)^{1 / p} \leq c\|g\|_{H_{L}^{p}} .
\end{aligned}
$$

This proves (i). 
Let us prove (ii). Note that by (b) of Lemma 3.4, for every $h_{t}(x) \in T_{2}^{p}$,

$$
\mathcal{R}\left(h_{t}\right)(x)=c_{m, s} \int_{0}^{\infty} Q_{s, t^{m}}\left(I-P_{s_{0}, t^{m}}\right)\left(h_{t}\right)(x) \frac{d t}{t} \in H_{L}^{p},
$$

where $c_{m, s}$ is a constant in (3.7). Therefore, for each continuous linear functional $\ell$ on the $H_{L}^{p}\left(\mathbb{R}^{n}\right)$ space, we obtain

$\left|(\ell \circ \mathcal{R})\left(h_{t}\right)\right|=\left|\ell \circ \mathcal{R}\left(h_{t}\right)\right| \leq\|\ell\|_{H_{L}^{p} \rightarrow \mathbb{C}}\left\|\mathcal{R}\left(h_{t}\right)\right\|_{H_{L}^{p}} \leq\|\ell\|_{H_{L}^{p} \rightarrow \mathbb{C}}\|\mathcal{R}\|_{T_{2}^{p} \rightarrow H_{L}^{p}}\left\|h_{t} \mid\right\|_{T_{2}^{p}}$ for all $h_{t}(x) \in T_{2}^{p}$. It then follows from (c) of Proposition 3.2 that there exists a function $z_{t}(x) \in T_{2}^{p, \infty}$ such that

$$
(\ell \circ \mathcal{R})\left(h_{t}\right)=\int_{\mathbb{R}_{+}^{n+1}} z_{t}(x) h_{t}(x) \frac{d x d t}{t} .
$$

On the other hand, by (3.10) we have that for any $g \in H_{L}^{p}\left(\mathbb{R}^{n}\right) \cap L^{2}\left(\mathbb{R}^{n}\right)$,

$$
g(x)=c_{m, s} \int_{0}^{\infty} Q_{s, t^{m}}\left(I-P_{s_{0}, t^{m}}\right) Q_{t^{m}} g(x) \frac{d t}{t} .
$$

This shows that for each continuous linear functional $\ell$ on the $H_{L}^{p}\left(\mathbb{R}^{n}\right)$ space, we have that for all $g \in H_{L}^{p}\left(\mathbb{R}^{n}\right)$,

$$
\ell(g)=\lim _{k \rightarrow \infty} \ell\left(g_{k}\right)=\lim _{k \rightarrow \infty} \ell \circ \mathcal{R} \circ Q_{t^{m}}\left(g_{k}\right)=\ell \circ \mathcal{R} \circ Q_{t^{m}}(g),
$$

where $\left\{g_{k}\right\}_{k}$ is a family of functions satisfying $g_{k} \in H_{L}^{p}\left(\mathbb{R}^{n}\right) \cap L^{2}\left(\mathbb{R}^{n}\right)$ and $\lim _{k \rightarrow \infty} g_{k}=$ $g$. From (4.8) and (4.9), we have that for all $g \in H_{L}^{p}\left(\mathbb{R}^{n}\right)$, there exists a function $z_{t}(x) \in T_{2}^{p, \infty}$ such that

$$
\begin{aligned}
\ell(g) & =l \circ \mathcal{R} \circ Q_{t^{m}}(g) \\
& =\int_{\mathbb{R}_{+}^{n+1}} z_{t}(x) Q_{t^{m}} g(x) \frac{d x d t}{t} \\
& =\int_{\mathbb{R}^{n}}\left(\int_{0}^{\infty} Q_{t^{m}}^{*} z_{t}(x) \frac{d t}{t}\right) g(x) d x \\
& \stackrel{\text { def }}{=} \int_{\mathbb{R}^{n}} f(x) g(x) d x,
\end{aligned}
$$

where $f(x)=\int_{0}^{\infty} Q_{t^{m}}^{*} z_{t}(x) \frac{d t}{t}$.

We now prove that $f \in \mathfrak{L}_{L^{*}}\left(\frac{1}{p}-1,2, s_{0}\right)$. For any ball $B=B\left(x_{B}, r_{B}\right)$, it follows from (4.10) and Proposition 3.5 that we obtain

$$
\begin{aligned}
\left(\int_{B}\left|f-P_{s_{0}, r_{B}^{m}}^{*} f\right|^{2} d x\right)^{1 / 2} & =\sup _{\|g\|_{L^{2}(B)} \leq 1}\left|\int_{\mathbb{R}^{n}}\left(f(x)-P_{s_{0}, r_{B}^{m}}^{*} f(x)\right) g(x) d x\right| \\
& =\sup _{\|g\|_{L^{2}(B)} \leq 1}\left|\int_{\mathbb{R}^{n}} f(x)\left(I-P_{s_{0}, r_{B}^{m}}\right) g(x) d x\right| \\
& \leq \sup _{\|g\|_{L^{2}(B)} \leq 1}\left|\ell\left(\left(I-P_{s_{0}, r_{B}^{m}}\right) g\right)\right| \\
& \leq\left\|\ell \sup _{\|g\|_{L^{2}(B)} \leq 1}\right\|\left(I-P_{s_{0}, r_{B}^{m}}\right) g \|_{H_{L}^{p}} \\
& \leq c\|\ell\||B|^{\frac{1}{2}+\left(\frac{1}{p}-1\right)} .
\end{aligned}
$$




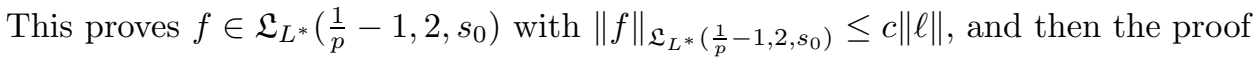
of Theorem 4.1 is complete.

As a consequence of Theorem 4.1, we have the following corollary.

Corollary 4.3. Suppose that the operator $L$ satisfies the assumptions (a) and (b) of Section 2. Let $0<\alpha<\theta(L) / n$ and $s \geq s_{0}=\left[\frac{n \alpha}{m}\right]$. Then the spaces $\mathfrak{L}_{L}(\alpha, 2, s)$ and $\mathfrak{L}_{L}\left(\alpha, 2, s_{0}\right)$ coincide, and their norms are equivalent.

Proof. Suppose $\frac{n}{n+\theta(L)}<p<1$ and $s \geq s_{0}=\left[\frac{n\left(\frac{1}{p}-1\right)}{m}\right]$. As in Definition 3.1, we define $H_{L^{*}}^{p, s}\left(\mathbb{R}^{n}\right)$ as the collection of all continuous linear functionals on $\mathfrak{L}_{L}\left(\frac{1}{p}-1,2, s\right)$ satisfying $Q_{t^{m}}^{*} f \in T_{2}^{p}$, and thus $H_{L^{*}}^{p}\left(\mathbb{R}^{n}\right)=H_{L^{*}}^{p, s_{0}}\left(\mathbb{R}^{n}\right)$. Similarly to the proof of Proposition 3.3, a molecular characterization (3.9) also holds for functions in $H_{L^{*}}^{p, s}\left(\mathbb{R}^{n}\right)$. Hence, the spaces $H_{L^{*}}^{p, s}\left(\mathbb{R}^{n}\right)$ and $H_{L^{*}}^{p}\left(\mathbb{R}^{n}\right)$ coincide, and their quasi-norms are equivalent. On the other hand, the same argument as in the proof of Theorem 4.1 shows that the dual space of $H_{L^{*}}^{p, s}\left(\mathbb{R}^{n}\right)$ is the space $\mathfrak{L}_{L}\left(\frac{1}{p}-1,2, s\right)$. For the proof, we omit details here. This, together with Theorem 4.1, gives Corollary 4.3 .

\section{Fractional integrals $L^{-\alpha}$ ON the Spaces $H_{L}^{p}\left(\mathbb{R}^{n}\right)$.}

We continue with the assumptions that the operator $L$ satisfies the assumptions (a) and (b) in Section 2.2. For $0<\alpha<\frac{n}{m}$, we consider the generalized fractional integrals $L^{-\alpha}$ associated with the operator $L$, defined by

$$
L^{-\alpha} f(x)=\frac{1}{\Gamma(\alpha)} \int_{0}^{\infty} t^{\alpha-1} e^{-t L} f(x) d t
$$

where $\Gamma(\alpha)$ is an appropriate constant. Note that if $L$ is the Laplacian $-\triangle$ on $\mathbb{R}^{n}$, then $L^{-\alpha}$ is the classical fractional integral. See, for example, Chapter 5 in 29 .

For $1<p<\infty$, we let $H_{L}^{p}\left(\mathbb{R}^{n}\right)=L^{p}\left(\mathbb{R}^{n}\right)$ (by (3.2)). The following theorem generalizes Theorem 4.1 of Taibleson-Weiss (34]).

Theorem 5.1. Suppose $\frac{n}{n+\theta(L)}<p_{1}<\infty, 0<\alpha<\frac{n}{m}$ and $\frac{1}{p_{2}}=\frac{1}{p_{1}}-\frac{m \alpha}{n}$, then the fractional integral $L^{-\alpha}$ maps $H_{L}^{p_{1}}\left(\mathbb{R}^{n}\right)$ continuously into $H_{L}^{p_{2}}$. If we replace $H_{L}^{\infty}\left(\mathbb{R}^{n}\right)$ with $\mathrm{BMO}_{L}\left(\mathbb{R}^{n}\right)$, the result holds for $\alpha=\frac{n}{m p_{1}}$.

Proof. For any given $p_{1}$, we let $\epsilon$ in (2.2) be the constant such that $\epsilon<\theta(L)$ and $\frac{n}{n+\epsilon}<p_{1}$. The result will follow from the repeated application four cases below.

Case I. $1<p_{1}<p_{2}<\infty$. This is a well-known result of Theorem II.2.7, page 12 of 36 .

Case II. $p_{1} \leq 1<p_{2}$ and $0<\alpha<\frac{n}{m}$. Choose $s_{0}=\left[\frac{n\left(\frac{1}{p_{1}}-1\right)}{m}\right]$ and $s \geq[\alpha+$ $\left.\frac{\epsilon}{m}\right]$. If $b(x)=\int_{0}^{\infty} Q_{s, t^{m}}\left(\mathcal{I}-P_{s_{0}, t^{m}}\right)(a(t, \cdot))(x) d t / t$, and $a(t, x)$ is a usual $T_{2}^{p_{1}}$-atom supported in $\widehat{B}$ (for some $B=B\left(z_{0}, r_{B}\right) \subset \mathbb{R}^{n}$ ), we will show that $\left\|L^{-\alpha}(b)\right\|_{p_{2}} \leq c$.

Case III. $p_{1}<p_{2} \leq 1$ and $0<\alpha<\frac{n}{m}$. If $b$ is a $\left(p_{1}, s\right)$-molecule as in Case II, we will show that $\left\|\mathcal{S}_{L}\left(L^{-\alpha}(b)\right)\right\|_{p_{2}} \leq c$. 
Case IV. $p>1$ and $\alpha=\frac{n}{m p}$. We need to show that $L^{-\frac{n}{m p}}: L^{p}\left(\mathbb{R}^{n}\right) \rightarrow$ $\mathrm{BMO}_{L}\left(\mathbb{R}^{n}\right)$ continuously. By Theorem 3.1 of $\left[18, \mathrm{BMO}_{L}\left(\mathbb{R}^{n}\right)=\left(H_{L^{*}}^{1}\left(\mathbb{R}^{n}\right)\right)^{\prime}\right.$ in which $L^{*}$ is the adjoint operator of $L$, and from Cases I and II we have the desired result by duality.

We give the details for Cases II and III.

Proof of Case II. Let $2<q_{2}<\infty$ such that $\frac{1}{p_{1}}-\frac{1}{p_{2}}=\frac{1}{2}-\frac{1}{q_{2}}=\frac{m \alpha}{n}$. We write

$$
\left\|L^{-\alpha}(b)\right\|_{p_{2}} \leq\left(\int_{4 B}\left|L^{-\alpha}(b)(x)\right|^{p_{2}} d x\right)^{1 / p_{2}}+\left(\int_{(4 B)^{c}}\left|L^{-\alpha}(b)(x)\right|^{p_{2}} d x\right)^{1 / p_{2}}=I+I I .
$$

Note that $\frac{1}{p_{1}}-\frac{1}{p_{2}}=\frac{1}{2}-\frac{1}{q_{2}}=\frac{m \alpha}{n}$. Using Hölder's inequality, Case I and (a) of Lemma 3.4, we obtain

$$
I \leq|B|^{\frac{1}{p_{2}}-\frac{1}{q_{2}}}\left\|L^{-\alpha}(b)\right\|_{q_{2}} \leq c|B|^{\frac{1}{p_{1}}-\frac{1}{2}}\|b\|_{2} \leq c|B|^{\frac{1}{p_{1}}-\frac{1}{2}}\left\||a \||_{T_{2}^{2}} \leq c .\right.
$$

We now estimate the term $I I$. We will show that there exists a constant $c>0$ such that for any $x \notin 4 B$,

$$
\left|L^{-\alpha}(b)(x)\right| \leq c r_{B}^{\epsilon+m \alpha+n-\frac{n}{p_{1}}}\left|x-z_{0}\right|^{-(n+\epsilon)} .
$$

Let us prove (5.2). For any $k=0, \cdots, s_{0}+1$, we denote by

$$
\Psi_{t, \nu}^{k}(L, s) f(x)=(-1)^{s+1}\left(t^{m}+(k+1) \nu^{m}\right)^{s+1}\left(\left.\frac{d^{s+1} P_{r}}{d r^{s+1}}\right|_{r=t^{m}+(k+1) \nu^{m}} f\right)(x) .
$$

Note that $\left(I-P_{s_{0}, t^{m}}\right) f(x)=\sum_{k=0}^{s_{0}+1}(-1)^{k} C_{s_{0}+1}^{k} e^{-k t^{m} L} f(x)$. This, together with property (2.10) and the fact that for $x \notin 4 B$ and $y \in B,|x-y|>2\left|x-z_{0}\right|$, yields

$$
\begin{aligned}
\left|L^{-\alpha}(b)(x)\right| \leq & c\left|\int_{0}^{\infty} \int_{0}^{\infty} t^{\alpha} e^{-t L} Q_{s, \nu^{m}}\left(\mathcal{I}-P_{s_{0}, \nu^{m}}\right) a(\nu, \cdot)(x) \frac{d \nu}{\nu} \frac{d t}{t}\right| \\
\leq & c \sum_{k=0}^{s_{0}+1} \mid \int_{0}^{\infty} \int_{0}^{\infty} \int_{B} \frac{\nu^{m(s+1)} t^{m \alpha}}{\left((k+1) \nu^{m}+t^{m}\right)^{s+1}} \\
& \times \Psi_{t, \nu}^{k}(L, s)(x, y) a(\nu, y) d y \frac{d \nu}{\nu} \frac{d t}{t} \mid \\
\leq & c \int_{0}^{\infty} \int_{0}^{\infty} \int_{B} \frac{\nu^{m(s+1)} t^{m \alpha}}{(\nu+t)^{m(s+1)}} \cdot \frac{(t+\nu)^{\epsilon}}{(t+\nu+|x-y|)^{n+\epsilon}}|a(\nu, y)| d y \frac{d \nu}{\nu} \frac{d t}{t} \\
\leq & c \int_{0}^{r_{B}} \int_{\widehat{B}} \frac{\nu^{m(s+1)} t^{m \alpha}}{(\nu+t)^{m(s+1)-\epsilon}}|a(\nu, y)| \frac{d y d \nu}{\nu} \frac{d t}{t}\left|x-z_{0}\right|^{-(n+\epsilon)} \\
& +c \int_{r_{B}}^{\infty} \int_{\widehat{B}} \frac{\nu^{m(s+1)} t^{m \alpha}}{(\nu+t)^{m(s+1)-\epsilon}}|a(\nu, y)| \frac{d y d \nu}{\nu} \frac{d t}{t}\left|x-z_{0}\right|^{-(n+\epsilon)} \\
= & I I_{1}+I I_{2} .
\end{aligned}
$$


We only estimate the term $I I_{2}$ since the estimate of the term $I I_{1}$ is much simpler. Using Hölder's inequality and the condition $m(s+1)>\epsilon+m \alpha$, we obtain

$$
\begin{aligned}
I I_{2} \leq & c|B|^{1 / 2} \int_{r_{B}}^{\infty}\left(\int_{0}^{r_{B}} \nu^{2(\epsilon+m \alpha)}\left(\frac{\nu}{t}\right)^{2(m(s+1)-\epsilon-m \alpha)} \frac{d \nu}{\nu}\right)^{1 / 2} \frac{d t}{t} \\
& \times\||| a\|||_{T_{2}^{2}}\left|x-z_{0}\right|^{-(n+\epsilon)} \\
\leq & c r_{B}^{m(s+1)+n-\frac{n}{p_{1}}}\left|x-z_{0}\right|^{-(n+\epsilon)} \int_{r_{B}}^{\infty} t^{\epsilon+m \alpha-m(s+1)} \frac{d t}{t} \\
\leq & c r_{B}^{\epsilon+m \alpha+n-\frac{n}{p_{1}}}\left|x-z_{0}\right|^{-(n+\epsilon)} .
\end{aligned}
$$

Similarly, we have that $I I_{1} \leq c r_{B}^{\epsilon+m \alpha+n-\frac{n}{p_{1}}}\left|x-z_{0}\right|^{-(n+\epsilon)}$, and then the estimate (5.2) is obtained. Hence,

$$
\begin{aligned}
\int_{(4 B)^{c}}\left|L^{-\alpha}(b)(x)\right|^{p_{2}} d x & \leq c r_{B}^{\left(\epsilon+m \alpha+n-\frac{n}{p_{1}}\right) p_{2}} \int_{(4 B)^{c}}\left|x-z_{0}\right|^{-(n+\epsilon) p_{2}} d x \\
& \leq c r_{B}^{m \alpha p_{2}+n-\frac{p_{2} n}{p_{1}}} \leq c .
\end{aligned}
$$

This completes the proof of Case II.

Proof of Case III. We write

$$
\begin{aligned}
\left\|\mathcal{S}_{L}\left(L^{-\alpha}(b)\right)\right\|_{p_{2}} \leq & c\left(\int_{4 B}\left|\mathcal{S}_{L}\left(L^{-\alpha}(b)\right)(x)\right|^{p_{2}} d x\right)^{1 / p_{2}} \\
& +c\left(\int_{(4 B)^{c}}\left|\mathcal{S}_{L}\left(L^{-\alpha}(b)\right)(x)\right|^{p_{2}} d x\right)^{1 / p_{2}} \\
= & I+I I .
\end{aligned}
$$

Since the area integral function $\mathcal{S}_{L}$ is bounded on $L^{r}$ for all $1<r<\infty$, by Case I we have $I \leq c|B|^{\frac{1}{p_{2}}-\frac{1}{q_{2}}}\left\|L^{-\alpha}(b)\right\|_{L^{q_{2}}} \leq c$.

We now estimate the term $I I$. As in Case II, it suffices to show that there exists a constant $c>0$ such that for any $x \notin 2 c_{1} B$,

$$
\mathcal{S}_{L}\left(L^{-\alpha}(b)\right)(x) \leq \operatorname{cr}_{B}^{\epsilon+m \alpha+n-\frac{n}{p_{1}}}\left|x-z_{0}\right|^{-(n+\epsilon)} .
$$

Let us prove (5.3). For any $k=0, \cdots, s_{0}+1$, we denote by

$$
\Psi_{t, \nu, \gamma}^{k}(L, s) f(x)=\left(t^{m}+\nu^{m}+(k+1) \gamma^{m}\right)^{s+2}\left(\left.\frac{d^{s+2} P_{r}}{d r^{s+2}}\right|_{r=t^{m}+\nu^{m}+(k+1) \gamma^{m}} f\right)(x)
$$

and

$$
h_{t, \nu, \gamma, k}^{m, \alpha, s}=\frac{t^{m} \nu^{m \alpha} \gamma^{m(s+1)}}{\left(t^{m}+\nu^{m}+(k+1) \gamma^{m}\right)^{s+2}} \leq c \frac{t^{m} \nu^{m \alpha} \gamma^{m(s+1)}}{(t+\nu+\gamma)^{m(s+2)}} .
$$

From estimate (2.10), we obtain that the kernel $\Psi_{t, \nu, \gamma}(L, s)(y, z)$ of the operator $\Psi_{t, \nu, \gamma}(L, s)$ satisfies

$$
\left|\Psi_{t, \nu, \gamma}^{k}(L, s)(y, z)\right| \leq c_{k} \frac{(t+\nu+\gamma)^{\epsilon}}{(t+\nu+\gamma+|y-z|)^{n+\epsilon}} .
$$


We then obtain

$$
\begin{aligned}
\mathcal{S}_{L}^{2}( & \left.L^{-\alpha}(b)\right)(x) \\
& \leq c\left(\mathcal{S}_{L}\left(\int_{0}^{\infty} \int_{0}^{\infty} \nu^{\alpha} e^{-\nu L} Q_{s, \gamma^{m}}\left(I-P_{s_{0}, \gamma^{m}}\right)^{s_{0}+1}(a(\gamma, \cdot)) \frac{d \nu}{\nu} \frac{d \gamma}{\gamma}\right)\right)^{2}(x) \\
& \leq \sum_{k=0}^{s_{0}+1} c_{k} \int_{\Gamma(x)}\left[\int_{0}^{\infty} \int_{0}^{r_{B}} h_{t, \nu, \gamma, k}^{m, \alpha, s} \Psi_{t, \nu, \gamma}^{k}(L, s)(a(\gamma, \cdot))(y) \frac{d \nu d \gamma}{\nu \gamma}\right]^{2} \frac{d y d t}{t^{n+1}} \\
& \leq c \int_{0}^{r_{B}} \int_{|y-x|<t} \\
& \times\left[\int_{0}^{r_{B}} \int_{\widehat{B}} \frac{t^{m} \nu^{m \alpha} \gamma^{m(s+1)}(t+\nu+\gamma)^{\epsilon}}{(t+\nu+\gamma)^{m(s+2)}(t+\nu+\gamma+|y-z|)^{n+\epsilon}}|a(\gamma, z)| \frac{d z d \nu d \gamma}{\nu \gamma}\right]^{2} \frac{d y d t}{t^{n+1}} \\
& +c \int_{0}^{r_{B}} \int_{|y-x|<t} \\
& \times\left[\int_{r_{B}}^{\infty} \int_{\widehat{B}} \frac{t^{m} \nu^{m \alpha} \gamma^{m(s+1)}(t+\nu+\gamma)^{\epsilon}}{(t+\nu+\gamma)^{m(s+2)}(t+\nu+\gamma+|y-z|)^{n+\epsilon}}|a(\gamma, z)| \frac{d z d \nu d \gamma}{\nu \gamma}\right]^{2} \frac{d y d t}{t^{n+1}} \\
& +c \int_{r_{B}}^{\infty} \int_{|y-x|<t} \\
& \times\left[\int_{0}^{r_{B}} \int_{\widehat{B}} \frac{t^{m} \nu^{m \alpha} \gamma^{m(s+1)}(t+\nu+\gamma)^{\epsilon}}{(t+\nu+\gamma)^{m(s+2)}(t+\nu+\gamma+|y-z|)^{n+\epsilon}}|a(\gamma, z)| \frac{d z d \nu d \gamma}{\nu \gamma}\right]^{2} \frac{d y d t}{t^{n+1}} \\
& \left.+c \int_{r_{B}}^{\infty} \int_{|y-x|<t}^{\infty}|a(\gamma, z)| \frac{d z d \nu d \gamma}{\nu \gamma}\right]^{2} \frac{d y d t}{t^{n+1}} \\
& \times\left[\int_{r_{B}}^{\infty} \int_{\widehat{B}} \frac{t^{m} \nu^{m \alpha} \gamma^{m(s+1)}(t+\nu+\gamma)^{\epsilon}}{(t+\nu+\gamma)^{m(s+2)}(t+\nu+\gamma+|y-z|)^{n+\epsilon}}\right. \\
& =I I_{1}+I I_{2}+I I_{3}+I I_{4} .
\end{aligned}
$$

Let us estimate the term $I I_{4}$. The same argument as in (3.13) shows that for $x \notin 4 B, t \geq r_{B}, z \in B_{2}$ and $|y-x|<t$, we have $(t+s+|y-z|) \geq c\left|x-z_{0}\right|$. Those, together with the fact that

$$
\frac{t^{m} \nu^{m \alpha} \gamma^{m(s+1)}(t+\nu+\gamma)^{\epsilon}}{(t+\nu+\gamma)^{m(s+2)}} \leq(t \nu)^{-\beta} \gamma^{\epsilon+m \alpha+2 \beta},
$$

where $\beta=(m(s+1)-m \alpha-\epsilon) / 2>0$, show that

$$
\begin{aligned}
I I_{4} \leq & c \int_{r_{B}}^{\infty}\left[\int_{r_{B}}^{\infty} \int_{\widehat{B}} \frac{1}{\nu^{\beta}} \gamma^{\epsilon+m \alpha+2 \beta}|a(\gamma, z)| \frac{d z d \nu d \gamma}{\nu \gamma}\right]^{2} \frac{d t}{t^{1+2 \beta}}\left|x-z_{0}\right|^{-2(n+\epsilon)} \\
\leq & c|B| \int_{r_{B}}^{\infty}\left[\int_{r_{B}}^{\infty}\left(\int_{0}^{r_{B}} \gamma^{2(\epsilon+m \alpha+2 \beta)} \frac{d \gamma}{\gamma}\right)^{1 / 2} \frac{d \nu}{\nu^{1+\beta}}\right]^{2} \frac{d t}{t^{1+2 \beta}} \|\left.|a|\right|_{T_{2}^{2}} ^{2} \\
& \times\left|x-z_{0}\right|^{-2(n+\epsilon)} \\
\leq & \operatorname{cr}_{B}^{2\left(n+\epsilon+m \alpha-\frac{n}{p_{1}}+2 \beta\right)} \int_{r_{B}}^{\infty}\left[\int_{r_{B}}^{\infty} \frac{d \nu}{\nu^{1+\beta}}\right]^{2} \frac{d t}{t^{1+2 \beta}}\left|x-z_{0}\right|^{-2(n+\epsilon)} \\
\leq & c r_{B}^{2(\epsilon+m \alpha)+2 n-\frac{2 n}{p_{1}}}\left|x-z_{0}\right|^{-2(n+\epsilon)} .
\end{aligned}
$$


The same argument as above shows that $I I_{1}+I I_{2}+I I_{3} \leq c r_{B}^{2(\epsilon+m \alpha)+2 n-\frac{2 n}{p_{1}}}$ $\times\left|x-z_{0}\right|^{-2(n+\epsilon)}$. This proves (5.3), and gives the desired estimate

$$
\int_{(4 B)^{c}}\left|\mathcal{S}_{L}\left(L^{-\alpha}(b)\right)(x)\right|^{p_{2}} d x \leq c .
$$

The proof of Case III is obtained. Hence, the proof of Theorem 5.1 is complete.

\section{Comparison between the Classical Hardy spaces $H^{p}\left(\mathbb{R}^{n}\right)$ AND THE SPACES $H_{L}^{p}\left(\mathbb{R}^{n}\right)$ ASSOCIATED WITH OPERATORS}

6.1. A sufficient condition for the $H_{L}^{p}\left(\mathbb{R}^{n}\right)$ spaces to coincide with the classical Hardy spaces. Assume that $L$ is a linear operator of type $\omega$ on $L^{2}\left(\mathbb{R}^{n}\right)$ with $\omega<\pi / 2$, hence $(-L)$ generates an analytic semigroup $e^{-z L}, 0 \leq|\operatorname{Arg}(z)|<$ $\pi / 2-\omega$. We assume that for each $t>0$, the kernel $p_{t}(x, y)$ of $e^{-t \bar{L}}$ is a Hölder continuous function in $x, y$ and there exist positive constants $m$ and $0<\gamma \leq 1$ such that for all $t>0$, and $x, y, h \in \mathbb{R}^{n}$,

$$
\left|p_{t}(x+h, y)-p_{t}(x, y)\right|+\left|p_{t}(x, y+h)-p_{t}(x, y)\right| \leq c|h|^{\gamma} \frac{t^{1 / m}}{\left(t^{1 / m}+|x-y|\right)^{n+1+\gamma}}
$$

whenever $2|h| \leq t^{1 / m}+|x-y|$; and

$$
\int_{\mathbb{R}^{n}} p_{t}(x, y) d x=\int_{\mathbb{R}^{n}} p_{t}(x, y) d y=1, \quad \forall t>0
$$

We have the following equivalence between the $H^{p}\left(\mathbb{R}^{n}\right)$ spaces and the $H_{L}^{p}\left(\mathbb{R}^{n}\right)$ spaces associated with operators.

Theorem 6.1. Assume that $L$ satisfies the assumptions (6.1), (6.2) and (6.3). Then for $\frac{n}{n+\gamma}<p \leq 1$, the spaces $H^{p}\left(\mathbb{R}^{n}\right)$ and $H_{L}^{p}\left(\mathbb{R}^{n}\right)$ coincide, and their quasinorms are equivalent.

As a consequence, for $0<\alpha<\frac{\gamma}{n}$, the classical Morrey-Campanato spaces $\mathrm{L}(\alpha, 2,0)$ and the spaces $\mathfrak{L}_{L}(\alpha, 2,0)$ coincide, and their norms are equivalent.

Proof. We remark that for $L$ satisfying (6.1), (6.2) and (6.3), our proof below shows that $L$ has a bounded holomorphic functional calculus on $L^{2}$ because the area integral functions $\mathcal{S}_{L}$ and $\mathcal{S}_{L^{*}}$ are bounded on $L^{2}$ in which $L^{*}$ is the adjoint operator of $L$. See Theorem 3 of [28].

Let $q_{t}(x, y)$ denote the kernel of the operator $Q_{t}=t \frac{d}{d t} e^{-t L}$. From Lemma 6.10 of [18, we have the following estimates: for any $0<\gamma_{1}<\gamma$ and $0<\beta_{1}<1$, there exist constant $c>0$ such that for all $t>0$, and $x, y, h \in \mathbb{R}^{n}$,

$$
\begin{gathered}
\left|q_{t}(x, y)\right| \leq c \frac{t^{\beta_{1} / m}}{\left(t^{1 / m}+|x-y|\right)^{n+\beta_{1}}}, \\
\left|q_{t}(x+h, y)-q_{t}(x, y)\right|+\left|q_{t}(x, y+h)-q_{t}(x, y)\right| \leq c|h|^{\gamma_{1}} \frac{t^{\beta_{1} / m}}{\left(t^{1 / m}+|x-y|\right)^{n+\beta_{1}+\gamma_{1}}}
\end{gathered}
$$

whenever $2|h| \leq t^{1 / m}+|x-y|$; and

$$
\int_{\mathbb{R}^{n}} q_{t}(x, y) d x=\int_{\mathbb{R}^{n}} q_{t}(x, y) d y=0, \quad \forall t>0 .
$$


It then follows from a standard harmonic analysis argument that $H_{L}^{p}\left(\mathbb{R}^{n}\right)=$ $H^{p}\left(\mathbb{R}^{n}\right)$ for $\frac{n}{n+\gamma}<p \leq 1$. See, for example, Chapter XIV, in 33. Hence, the proof of Theorem 6.1 is complete.

Remark. As noted before, the assumptions (6.1), 6.2 and (6.3) are satisfied for the divergence form operator $L=-\operatorname{div} A \nabla$ when $A$ has real entries, or when the dimension $n=1$ or 2 in the case of complex entries. See Chapter 1 of [4] and [2].

6.2. An example of $H_{L}^{p}\left(\mathbb{R}^{n}\right) \varsubsetneqq H^{p}\left(\mathbb{R}^{n}\right)$. To begin with, let us recall some basic facts about the Neumann Laplacian $\triangle_{N}$ on $\mathbb{R}^{n}$, which was studied in 12 . In what follows, $\mathbb{R}_{+}^{n}$ denotes the upper-half space in $\mathbb{R}^{n}$, i.e.,

$$
\mathbb{R}_{+}^{n}=\left\{\left(x^{\prime}, x_{n}\right) \mid \in \mathbb{R}^{n}: x^{\prime}=\left(x_{1}, \cdots, x_{n-1}\right) \in \mathbb{R}^{n-1}, x_{n}>0\right\} .
$$

Similarly, $\mathbb{R}_{-}^{n}$ denotes the lower-half space in $\mathbb{R}^{n}$.

We denote by $\triangle_{N_{+}}$(resp. $\triangle_{N_{-}}$) the Neumann Laplacian on $\mathbb{R}_{+}^{n}$ (resp. on $\mathbb{R}_{-}^{n}$ ). See page 57 of 31 . The Neumann Laplacians are self-adjoint and positive definite operators. Using the spectral theory one can define the semigroup $\left\{\exp \left(-t \triangle_{N_{+}}\right)\right\}_{t \geq 0}\left(\operatorname{resp} .\left\{\exp \left(-t \triangle_{N_{-}}\right)\right\}_{t \geq 0}\right)$ generated by the operator $\triangle_{N_{+}}$(resp. $\left.\triangle_{N_{-}}\right)$. For any $f$ defined on $\mathbb{R}^{n}$, we set

$$
f_{-}=\left.f\right|_{\mathbb{R}_{-}^{n}} \text { and } f_{+}=\left.f\right|_{\mathbb{R}_{+}^{n}}
$$

where $\left.f\right|_{\mathbb{R}_{+}^{n}}$ and $\left.f\right|_{\mathbb{R}_{-}^{n}}$ are restrictions of the function $f$ to $\mathbb{R}_{+}^{n}$ and $\mathbb{R}_{-}^{n}$, respectively. We let $\triangle_{N}$ be the uniquely determined unbounded operator acting on $L^{2}\left(\mathbb{R}^{n}\right)$ such that

$$
\left(\triangle_{N} f\right)_{+}=\triangle_{N_{+}} f_{+} \quad \text { and } \quad\left(\triangle_{N} f\right)_{-}=\triangle_{N_{-}} f_{-}
$$

for all $f: \mathbb{R}^{n} \mapsto \mathbb{R}$ such that $f_{+} \in W^{1,2}\left(\mathbb{R}_{+}^{n}\right)$ and $f_{-} \in W^{1,2}\left(\mathbb{R}_{-}^{n}\right)$. Then, $\triangle_{N}$ is a positive definite self-adjoint operator and let $p_{t}(x, y)$ be the heat kernel of the semigroup $\exp \left(-t \triangle_{N}\right)$. By (6.4), we have

$$
\left(\exp \left(-t \triangle_{N}\right) f\right)_{+}=\exp \left(-t \triangle_{N_{+}}\right) f_{+} \text {and }\left(\exp \left(-t \triangle_{N}\right) f\right)_{-}=\exp \left(-t \triangle_{N_{-}}\right) f_{-} .
$$

Moreover, we have

$$
p_{t}(x, y)=\frac{1}{(4 \pi t)^{\frac{n}{2}}} e^{-\frac{\left|x^{\prime}-y^{\prime}\right|^{2}}{4 t}}\left(e^{-\frac{\left|x_{n}-y_{n}\right|^{2}}{4 t}}+e^{-\frac{\left|x_{n}+y_{n}\right|^{2}}{4 t}}\right) H\left(x_{n} y_{n}\right),
$$

where $H: \mathbb{R} \rightarrow\{0,1\}$ is the Heaviside function, given by $H(t)=0$ if $t<0 ; 1$ if $t \geq 0$. See Section 2 of 12 .

The aim of this section is to prove the following proposition.

Proposition 6.2. Suppose $\frac{n}{n+1}<p \leq 1$. The operator $\triangle_{N}$ satisfies the assumptions (a) and (b) of Section 2. Moreover, we have $H_{\triangle_{N}}^{p}\left(\mathbb{R}^{n}\right) \varsubsetneqq H^{p}\left(\mathbb{R}^{n}\right)$. That is, $H_{\triangle_{N}}^{p}\left(\mathbb{R}^{n}\right)$ is a proper subspace of the classical $H^{p}\left(\mathbb{R}^{n}\right)$ space.

Proof. Since $\triangle_{N}$ is a self-adjoint positive definite operator, hence it has a bounded $H_{\infty}$-calculus in $L^{2}\left(\mathbb{R}^{n}\right)$. From the equation (6.5), $\triangle_{N}$ generates the conservative semigroup $e^{-t \triangle_{N}}$ that is $e^{-t \triangle_{N}}(1)=1$ for all $t>0$, which satisfies the assumptions (a) and (b) of Section 2. This gives that

$$
H_{\Delta_{N}}^{p}\left(\mathbb{R}^{n}\right) \subseteq H^{p}\left(\mathbb{R}^{n}\right), \quad \frac{n}{n+1}<p \leq 1 .
$$


On the other hand, from Theorem 4.1 of [12] and Proposition 5.3 of [19], this operator $\triangle_{N}$ generates the spaces $\mathfrak{L}_{\triangle_{N}}(\alpha, 2,0)$ with $0 \leq \alpha<n^{-1}$ such that

(i) $\mathrm{L}(\alpha, 2,0) \subseteq \mathfrak{L}_{\triangle_{N}}(\alpha, 2,0)$.

(ii) We have $f(x)=\log |x| \chi_{\left\{x: x \in \mathbb{R}_{+}^{n}\right\}}(x) \in \mathrm{BMO}_{\triangle_{N}}\left(\mathbb{R}^{n}\right)$, however, $f \notin \operatorname{BMO}\left(\mathbb{R}^{n}\right)$.

(iii) For $0<\alpha<n^{-1}$, we have $f(x)=e^{-|x|^{2}} \chi_{\left\{x: x \in \mathbb{R}_{+}^{n}\right\}}(x) \in \mathfrak{L}_{\triangle_{N}}(\alpha, 2,0)$, however, $f \notin \mathrm{L}(\alpha, 2,0)$.

From the properties (i), (ii) and (iii) above, we have that $\mathfrak{L}(\alpha, 2,0)$ is a proper subspace of $\mathfrak{L}_{\triangle_{N}}(\alpha, 2,0)$. Proposition 6.2 then follows from Theorem 4.1 and the fact that $\left(H^{p}\right)^{\prime}=\mathrm{L}\left(\frac{1}{p}-1,2,0\right)$ (see, for example, Theorem 2.7 of 34 ).

Note. In [37, it was asked if a proper subspace of the classical Hardy space $H^{p}\left(\mathbb{R}^{n}\right)$ for $p \leq 1$ exists in which the subspace is characterized by maximal functions. For $p=1$, this question was answered positively in 35 . Our Proposition 6.2 gives a proper subspace of the classical Hardy space $H^{p}\left(\mathbb{R}^{n}\right)$ for $\frac{n}{n+1}<p \leq 1$ where the subspace is characterized by area integral functions.

\section{ACKNOWLEDGMENTS}

The author would like to thank X.T. Duong and S. Mayboroda for helpful suggestions and also the referee for helpful comments.

\section{REFERENCES}

[1] D. Albrecht, X.T. Duong and A. McIntosh, Operator theory and harmonic analysis, Instructional Workshop on Analysis and Geometry (Proc. Centre Math. Analysis, 34, A.N.U., Canberra, 1996) pp 77-136. MR1394696 (97e:47001)

[2] P. Auscher and E. Russ, Hardy spaces and divergence operators on strongly Lipschitz domain of $\mathbb{R}^{n}$, J. Funct. Anal., 201 (2003), 148-184. MR1986158 (2004c:42049)

[3] P. Auscher and P. Tchamitchian, Calcul fonctionnel précisé pour des opérateurs elliptiques complexes en dimension un (et applications à certaines équations elliptiques complexes en dimension deux), Ann. Institut Fourier (Grenoble), 45 (1995), 721-778. MR.1340951(96f:35036)

[4] P. Auscher and P. Tchamitchian, Square root problem for divergence operators and related topics, Asterisque, 249, Soc. Math. France, 1998. MR.1651262 (2000c:47092)

[5] P. Auscher, X.T. Duong and A. McIntosh, Boundedness of Banach space valued singular integral operators and Hardy spaces, unpublished preprint, (2005).

[6] S. Blunck and P.C. Kunstmann, Weak type $(p, p)$ estimates for Riesz transforms, Math. Z., 247 (2004), 137-148. MR2054523 (2005f:35071)

[7] T. Coulhon and X.T. Duong, Maximal regularity and kernel bounds: observations on a theorem by Hieber and Prüss, Adv. Differential Equations, 5 (2000), 343-368. MR 1734546 (2001d:34087)

[8] D-C. Chang, S.G. Krantz and E.M. Stein, $H^{p}$ theory on a smooth domain in $\mathbb{R}^{n}$ and elliptic boundary value problems, J. Funct. Anal., 114 (1993), 286-347. MR1223705 (94j:46032)

[9] R.R. Coifman, Y. Meyer and E.M. Stein, Un nouvel espace adapté a l'étude des opérateurs définis par des intégrales singuliéres, in "Proc. Conf. Harmonic Analysis, Cortona", Lecture Notes in Math. Vol. 992, pp, 1-15, Springer-Verlag, Berlin/New York, 1983. MR729344 (85j:42032)

[10] R.R. Coifman, Y. Meyer and E.M. Stein, Some new functions and their applications to harmonic analysis, J. Funct. Analysis, 62 (1985), 304-315. MR791851 (86i:46029)

[11] E.B. Davies, Heat kernels and spectral theory, Cambridge Univ. Press, 1989. MR990239 (90e:35123)

[12] D.G. Deng, X.T. Duong, A. Sikora and L.X. Yan, Comparison of the classical BMO with the BMO spaces associated with operators and applications, to appear, Rev. Mat. Iberoamericana (2008). 
[13] D.G. Deng, X.T. Duong and L.X. Yan, A characterization of the Morrey-Campanato spaces, Math. Z., 250 (2005), 641-655. MR2179615 (2006g:42039)

[14] X.T. Duong and A. McIntosh, Singular integral operators with non-smooth kernels on irregular domains, Rev. Mat. Iberoamericana, 15 (1999), 233-265. MR.1715407 (2001e:42017a)

[15] X.T. Duong, E.M. Ouhabaz and L.X. Yan, Endpoint estimates for Riesz transforms of magnetic Schrödinger operators, Arkiv för Matematik 44 (2006), 261-275. MR2292721 (2008a:35224)

[16] P.L. Duren, B.W. Romberg and A.L. Shields, Linear functionals on $H^{p}$ spaces with $0<p<1$, J. Reine Angew. Math., 238 (1969), 32-60. MR0259579 (41:4217)

[17] X.T. Duong and L.X. Yan, New function spaces of BMO type, the John-Nirenberg inequality, interpolation and applications, Comm. Pure Appl. Math., 58 (2005), 1375-1420. MR2162784 (2006i:26012)

[18] X.T. Duong and L.X. Yan, Duality of Hardy and BMO spaces associated with operators with heat kernel bounds, J. Amer. Math. Soc., 18 (2005), 943-973. MR2163867(2006d:42037)

[19] X.T. Duong and L.X. Yan, New Morrey-Campanato spaces associated with operators and applications, preprint, 2005.

[20] J. Dziubański and J. Zienkiewicz, $H^{p}$ spaces associated with Schrödinger operators with potentials from reverse Hölder classes, Colloq. Math., 98 (2003), 5-38. MR2032068 (2004k:42038)

[21] C. Fefferman and E.M. Stein, $H^{p}$ spaces of several variables, Acta Math., 129 (1972), 137195. MR0447953 (56:6263)

[22] F. John and L. Nirenberg, On functions of bounded mean oscillation. Comm. Pure Appl. Math., 14 (1961), 415-426. MR0131498(24:A1348)

[23] S. Janson, M.H. Taibleson, G. Weiss, Elementary characterizations of the Morrey-Campanato spaces, Lecture Notes in Math., 992 (1983), 101-114. MR.729349 (85k:46033)

[24] S. Hofmann and J.M. Martell, $L^{p}$ bounds for Riesz transforms and square roots associated to second order elliptic operators, Publ. Mat., 47 (2003), 497-515. MR2006497(2004i:35067)

[25] J.M. Martell, Sharp maximal functions associated with approximations of the identity in spaces of homogeneous type and applications, Studia Math., 161(2004), 113-145. MR2033231 (2005b:42016)

[26] A. McIntosh, Operators which have an $H_{\infty}$-calculus, Miniconference on operator theory and partial differential equations (Proc. Centre Math. Analysis, ANU, Canberra 14, 1986), 210231. MR.912940 (88k:47019)

[27] E.M. Ouhabaz, Analysis of heat equations on domains. London Math. Soc. Mono. 31, Princeton Univ. Press, (2004). MR2124040 (2005m:35001)

[28] S. Semmes, Square function estimates and the T(b) theorem, Proc. Amer. Math. Soc., 110 (1990), 721-726. MR1028049 (91h:42018)

[29] E.M. Stein, Singular integral and differentiability properties of functions, Princeton Univ. Press, 30, (1970). MR0290095 (44:7280)

[30] E.M. Stein, Harmonic analysis: Real variable methods, orthogonality and oscillatory integrals, Princeton Univ. Press, Princeton, NJ, 1993. MR1232192 (95c:42002)

[31] W.A. Strauss, Partial differential equations: An introduction. John Wiley \& Sons, Inc., New York, 1992. MR:1159712 (92m:35001)

[32] E.M. Stein and G. Weiss, On the theory of harmonic functions of several variables I, The theory of $H^{p}$ spaces, Acta Math., 103 (1960), 25-62. MR.0121579(22:12315)

[33] A. Torchinsky, Real-variable methods in harmonic analysis, Pure and Applied Math., Vol 123, Academic Press, (1986). MR869816 (88e:42001)

[34] M.W. Taibleson and G. Weiss, The molecular characterization of certain Hardy spaces, Astérisque. 77, 68-149 (1980). MR0604370(83g:42012)

[35] A. Uchiyama and J.M. Wilson, Approximate identities and $H^{1}(\mathbb{R})$, Proc. Amer. Math. Soc., 88 (1983), 53-58. MR691278 (84c:42032)

[36] N. Varopoulos, L. Saloff-Coste and T. Coulhon, Analysis and geometry on groups. Cambridge Univ. Press, London, 1993. MR1218884 (95f:43008)

[37] G. Weiss, Some problems in the theory of Hardy spaces, Proc. Sympos. Pure Math. Vol. 35, Amer. Math. Soc., Providence, R.I., (1979), 189-200. MR545258 (80j:30051) 
[38] L.X. Yan, Littlewood-Paley functions associated to second order elliptic operators, Math. Z., 246, (2004), 655-666. MR.2045834 (2005a:42015)

[39] K. Yosida, Functional Analysis (Fifth edition), Springer-Verlag, Berlin, 1978. MR0500055 $(58: 17765)$

Department of Mathematics, Zhongshan University, Guangzhou, 510275, People's RePUBLIC OF CHINA

E-mail address: mcsylx@mail.sysu.edu.cn 